\title{
Invariant plurisubharmonic functions on non-compact Hermitian symmetric spaces
}

\author{
Laura Geatti ${ }^{1} \cdot$ Andrea lannuzzi $^{1}$
}

Received: 8 February 2020 / Accepted: 23 February 2021 / Published online: 27 May 2021

(c) The Author(s) 2021

\begin{abstract}
Let $G / K$ be an irreducible non-compact Hermitian symmetric space and let $D$ be a $K$ invariant domain in $G / K$. In this paper we characterize several classes of $K$-invariant plurisubharmonic functions on $D$ in terms of their restrictions to a slice intersecting all $K$-orbits. As applications we show that $K$-invariant plurisubharmonic functions on $D$ are necessarily continuous and we reproduce the classification of Stein $K$-invariant domains in $G / K$ obtained by Bedford and Dadok. (J Geom Anal 1:1-17, 1991).
\end{abstract}

Keywords Hermitian symmetric spaces $\cdot$ Stein domains $\cdot$ Plurisubharmonic functions

Mathematics Subject Classification 32M15 $\cdot 31 \mathrm{C} 10 \cdot 32 \mathrm{~T} 05$

\section{Introduction}

Let $G / K$ be an irreducible non-compact Hermitian symmetric space of rank $r$. By the polydisk theorem the space $G / K$ contains a closed subspace $\Delta^{r}$, biholomorphic to an $r$ dimensional polydisk, with the property that $G / K=K \cdot \Delta^{r}$. If $D$ is a $K$-invariant domain in $G / K$, then $D=K \cdot R$, where $R:=D \cap \Delta^{r}$ is a Reinhardt domain in $\Delta^{r}$. The polydisk $\Delta^{r}$ and $R$ are invariant under the group $T \ltimes \mathcal{S}_{r}$, generated by rotations and coordinate permutations.

As the Reinhardt domain $R$ intersects all the $K$-orbits in $D$, it encodes all information on the $K$-invariant objects in $D$. In this paper we focus on the $K$-invariant plurisubhar-

The authors acknowledge the MIUR Excellence Department Project awarded to the Department of Mathematics, University of Rome "Tor Vergata", CUP E83C18000100006. This research was partially supported by GNSAGA-INDAM.

Laura Geatti

geatti@mat.uniroma2.it

Andrea Iannuzzi

iannuzzi@mat.uniroma2.it

1 Dipartimento di Matematica, Università di Roma “Tor Vergata”, Via della Ricerca Scientifica 1, 00133 Rome, Italy 
monic functions. When $D$ is Stein, we obtain the following characterization of the class $P^{\infty,+}(D)^{K}$ of smooth, $K$-invariant, strictly plurisubharmonic functions on $D$ :

$$
f \in P^{\infty,+}(D)^{K} \text { if and only if }\left.f\right|_{R} \in P^{\infty,+}(R)^{T \ltimes \mathcal{S}_{r}},
$$

where $\left.f\right|_{R}$ is the restriction of $f$ to $R$. Such result is later extended to wider classes of plurisubharmonic functions as follows. Let $P^{\infty}(D)^{K}$ denote the class of smooth, $K$ invariant, plurisubharmonic functions and $P^{+}(D)^{K}$ (resp. $P(D)^{K}$ ) the class of $K$-invariant, strictly plurisubharmonic (resp. plurisubharmonic) functions on $D$. One has:

Theorem 4.13 The restriction map $\left.f \rightarrow f\right|_{R}$ is a bijection between

(i) $P^{\infty,+}(D)^{K}$ and $P^{\infty,+}(R)^{T \ltimes \mathcal{S}_{r}}$,

(ii) $P(D)^{K}$ and $P(R)^{T \ltimes \mathcal{S}_{r}}$,

(iii) $P^{\infty}(D)^{K}$ and $P^{\infty}(R)^{T \ltimes \mathcal{S}_{r}}$,

(iv) $P^{+}(D)^{K}$ and $P^{+}(R)^{T \ltimes \mathcal{S}_{r}}$.

As a by-product we reproduce the classification of Stein $K$-invariant domains in $G / K$ obtained by Bedford and Dadok in some classical cases by direct computations [2] (see also [5] for related results).

Corollary 4.8 Let $D$ be a $K$-invariant domain in $G / K$.

(i) If $G / K$ is of tube type, then $D$ is Stein if and only if $R$ is Stein and connected.

(ii) If $G / K$ is not of tube type, then $D$ is Stein if and only if $R$ is Stein and complete. In particular $R$ contains the origin and it is connected.

The proof of our results is carried out as follows. Let $\mathfrak{g}=\mathfrak{k} \oplus \mathfrak{p}$ be a Cartan decomposition of the Lie algebra $\mathfrak{g}$ of $G$, let $\mathfrak{a}$ be a maximal abelian subspace of $\mathfrak{p}$, with Weyl group $W$, and let $G=K \exp \mathfrak{a} K$ be the corresponding decomposition of $G$. Every $K$-invariant domain $D$ in $G / K$ is uniquely determined by a $W$-invariant domain $\mathcal{D}_{\mathfrak{a}}$ in $\mathfrak{a}$ by

$$
D=K \exp \mathcal{D}_{\mathfrak{a}} K / K
$$

Similarly, every smooth $K$-invariant function $f$ on $D$ is uniquely determined by the smooth $W$-invariant function $\tilde{f}$ on $\mathcal{D}_{\mathfrak{a}}$ defined by

$$
\tilde{f}(H):=f(\exp (H) K), \text { for } H \in \mathcal{D}_{\mathfrak{a}},
$$

(cf. $[4,6])$.

As a first step we explicitly express the Levi form of $f$ in terms of the first and second derivatives of $\widetilde{f}$. This is achieved in Proposition 3.1 by means of a fine decomposition of the tangent bundle of $D$, induced by the restricted root decomposition of $\mathfrak{g}$, and a simple pluripotential argument which enable us to maximally exploit the symmetries at hand.

The Levi form computation is a key ingredient for our results. It leads to the following characterization of smooth $K$-invariant strictly plurisubharmonic functions on a Stein $K$ invariant domain $D$ (Theorem 4.5$)$ :

$$
f \in P^{\infty,+}(D)^{K} \quad \text { if and only if } \quad \tilde{f} \in \log \operatorname{Con} v^{\infty,+}\left(\mathcal{D}_{\mathfrak{a}}\right)^{W},
$$

where the latter class consists of smooth $W$-invariant functions on $\mathcal{D}_{\mathfrak{a}}$ satisfying the appropriate differential positivity condition. We also show that $\tilde{f}$ belongs to $\log \operatorname{Conv} v^{\infty,+}\left(\mathcal{D}_{\mathfrak{a}}\right)^{W}$ if and only if the corresponding $T \ltimes \mathcal{S}_{r}$-invariant function on the associated Reinhardt domain $R$ is smooth and strictly plurisubharmonic.

This fact, which may be of independent interest in the context of Reinhardt domains, implies (i) in the above theorem. When extending such characterization to the non-smooth 
setting (Theorem 4.12), it turns out that the $K$-invariant plurisubharmonic functions on $D$ are necessarily continuous.

In the appendix we explicitly determine a $K$-invariant potential of the Killing metric on $G / K$ in a Lie theoretical fashion (Proposition 5.1) and we observe that, up to an additive constant, it coincides with the logarithm of the Bergman kernel function.

Finally, we point out that our methods require no classification results, nor any distinction between classical and exceptional cases.

We wish to thank our colleague Stefano Trapani for several useful discussions and suggestions.

\section{Preliminaries}

Let $\mathfrak{g}$ be a non-compact semisimple Lie algebra and let $\mathfrak{k}$ be a maximal compact subalgebra of $\mathfrak{g}$. Let $\mathfrak{g}=\mathfrak{k} \oplus \mathfrak{p}$ be the Cartan decomposition of $\mathfrak{g}$ with respect to $\mathfrak{k}$, with Cartan involution $\theta$. Let $\mathfrak{a}$ be a maximal abelian subspace in $\mathfrak{p}$. The dimension $r$ of $\mathfrak{a}$ is by definition the rank of $G / K$. Let $\mathfrak{g}=\mathfrak{m} \oplus \mathfrak{a} \oplus \bigoplus_{\alpha \in \Sigma} \mathfrak{g}^{\alpha}$ be the restricted root decomposition of $\mathfrak{g}$, where $\mathfrak{m}$ is the centralizer of $\mathfrak{a}$ in $\mathfrak{k}$, the joint eigenspace $\mathfrak{g}^{\alpha}=\{X \in \mathfrak{g} \mid[H, X]=\alpha(H) X$, for all $H \in \mathfrak{a}\}$ is the $\alpha$-restricted root space and the restricted root system $\Sigma$ consists of those $\alpha \in \mathfrak{a}^{*}$ for which $\mathfrak{g}^{\alpha} \neq\{0\}$. Denote by $B(\cdot, \cdot)$ the Killing form of $\mathfrak{g}$, as well as its holomorphic extension to $\mathfrak{g}^{\mathbb{C}}$ (which coincides with the Killing form of $\mathfrak{g}^{\mathbb{C}}$ ).

For $\alpha \in \Sigma$, consider the $\theta$-stable space $\mathfrak{g}[\alpha]:=\mathfrak{g}^{\alpha} \oplus \mathfrak{g}^{-\alpha}$, and denote by $\mathfrak{k}[\alpha]$ and $\mathfrak{p}[\alpha]$ the projections of $\mathfrak{g}[\alpha]$ along $\mathfrak{p}$ and $\mathfrak{k}$, respectively. Let $\Sigma^{+}$be a choice of positive roots in $\Sigma$. Then

$$
\mathfrak{k}=\mathfrak{m} \oplus \bigoplus_{\alpha \in \Sigma^{+}} \mathfrak{k}[\alpha] \quad \text { and } \quad \mathfrak{p}=\mathfrak{a} \oplus \bigoplus_{\alpha \in \Sigma^{+}} \mathfrak{p}[\alpha]
$$

are $B$-orthogonal decompositions of $\mathfrak{k}$ and $\mathfrak{p}$, respectively.

Lemma 2.1 Every element $X$ in $\mathfrak{p}$ decomposes in a unique way as

$$
X_{\mathfrak{a}}+\sum_{\alpha \in \Sigma^{+}} P^{\alpha}
$$

where $X_{\mathfrak{a}} \in \mathfrak{a}$ and $P^{\alpha} \in \mathfrak{p}[\alpha]$. The vector $P^{\alpha}$ can be written uniquelyas $P^{\alpha}=X^{\alpha}-\theta X^{\alpha}$, where $X^{\alpha}$ is the component of $X$ in the root space $\mathfrak{g}^{\alpha}$. Moreover, $\left[H, P^{\alpha}\right]=\alpha(H) K^{\alpha}$, where $K^{\alpha}$ is the element in $\mathfrak{k}[\alpha]$ defined by $K^{\alpha}=X^{\alpha}+\theta X^{\alpha}$.

Proof By the restricted root decomposition, every $X \in \mathfrak{g}$ can be written as

$$
X=X_{\mathfrak{m}}+X_{\mathfrak{a}}+\sum_{\alpha \in \Sigma^{+}} X^{\alpha}+X^{-\alpha} .
$$

Then $X \in \mathfrak{p}$ if and only if $X_{\mathfrak{m}}=0$ and $\theta\left(X^{\alpha}+X^{-\alpha}\right)=-\left(X^{\alpha}+X^{-\alpha}\right)$, for all $\alpha \in \Sigma$. In particular $\theta X^{\alpha}=-X^{-\alpha}$, and the lemma follows.

The restricted root system of a simple Lie algebra $\mathfrak{g}$ of Hermitian type is either of type $C_{r}$ (if $G / K$ is of tube type) or of type $B C_{r}$ (if $G / K$ is not of tube type), i.e. there exists a basis $\left\{e_{1}, \ldots, e_{r}\right\}$ of $\mathfrak{a}^{*}$ for which

$$
\begin{aligned}
& \Sigma^{+}=\left\{2 e_{j}, 1 \leq j \leq r, \quad e_{k} \pm e_{l}, 1 \leq k<l \leq r\right\}, \quad \text { for type } C_{r}, \\
& \Sigma^{+}=\left\{e_{j}, 2 e_{j}, 1 \leq j \leq r, \quad e_{k} \pm e_{l}, \quad 1 \leq k<l \leq r\right\}, \quad \text { for type } B C_{r} .
\end{aligned}
$$


With such a choice of a positive system $\Sigma^{+}$, the roots

$$
2 e_{1}, \ldots, 2 e_{r}
$$

form a maximal set of long strongly orthogonal positive restricted roots, i.e. such that $2 e_{k} \pm$ $2 e_{l} \notin \Sigma$, for $k \neq l$.

For $j=1, \ldots, r$, the root spaces $\mathfrak{g}^{2 e_{j}}$ are one-dimensional. Choose generators $E^{j} \in \mathfrak{g}^{2 e_{j}}$ such that the $\mathfrak{s l}(2)$-triples $\left\{E^{j}, \theta E^{j}, A_{j}:=\left[\theta E^{j}, E^{j}\right]\right\}$ are normalized as follows

$$
\left[A_{j}, E^{j}\right]=2 E^{j}, \quad \text { for } j=1, \ldots, r .
$$

Denote by $I_{0}$ the $G$-invariant complex structure of $G / K$. We also assume that $I_{0}\left(E^{j}-\right.$ $\left.\theta E^{j}\right)=A_{j}$ (see [7], Def.2.1). By the strong orthogonality of $2 e_{1}, \ldots, 2 e_{r}$, the vectors $A_{1}, \ldots, A_{r}$ form a $B$-orthogonal basis of $\mathfrak{a}$, dual to the basis $e_{1}, \ldots, e_{r}$ of $\mathfrak{a}^{*}$, and the associated $\mathfrak{s l}(2)$-triples pairwise commute. For $j=1, \ldots, r$, define

$$
K^{j}:=E^{j}+\theta E^{j} \quad \text { and } \quad P^{j}:=E^{j}-\theta E^{j} .
$$

Denote by $W$ the Weyl group of $\mathfrak{a}$, i.e. the quotient of the normalizer over the centralizer of $\mathfrak{a}$ in $K$. As $\mathfrak{g}$ is of Hermitian type, $W$ acts on $\mathfrak{a}$ by signed permutations of the coordinates determined by $A_{1}, \ldots, A_{r}$.

On $\mathfrak{p} \cong T_{e K} G / K$ the complex structure $I_{0}$ coincides with the adjoint action of the element $Z_{0} \in Z(\mathfrak{k})$ given by

$$
Z_{0}=S_{0}+\frac{1}{2} \sum_{j=1}^{r} K^{j},
$$

for some element $S_{0}$ in a Cartan subalgebra $\mathfrak{s}$ of $\mathfrak{m}$. In the tube case, one has $S_{0}=0$ (see [7], Lem. 2.2). The complex structure $I_{0}$ permutes the blocks of the decomposition (1) of $\mathfrak{p}$ (cf. [11]), namely

$$
I_{0} \mathfrak{a}=\bigoplus_{j=1}^{r} \mathfrak{p}\left[2 e_{j}\right], \quad I_{0} \mathfrak{p}\left[e_{j}+e_{l}\right]=\mathfrak{p}\left[e_{j}-e_{l}\right], \quad I_{0} \mathfrak{p}\left[e_{j}\right]=\mathfrak{p}\left[e_{j}\right] .
$$

The next lemma gives a more detailed description of the complex structure $I_{0}$ on $\mathfrak{p}$. In order to state it, we need to recall a few more facts. Let $\mathfrak{g}^{\mathbb{C}}=\mathfrak{h}^{\mathbb{C}} \oplus \bigoplus_{\mu \in \Delta} \mathfrak{g}^{\mu}$ be the root decomposition of $\mathfrak{g}^{\mathbb{C}}$ with respect to the maximally split Cartan subalgebra $\mathfrak{h}=\mathfrak{s} \oplus \mathfrak{a}$ of $\mathfrak{g}$. Let $\sigma$ be the conjugation of $\mathfrak{g}^{\mathbb{C}}$ with respect to $\mathfrak{g}$. Let $\theta$ denote also the $\mathbb{C}$-linear extension of $\theta$ to $\mathfrak{g}^{\mathbb{C}}$. One has $\theta \sigma=\sigma \theta$. Write $\bar{Z}:=\sigma Z$, for $Z \in \mathfrak{g}^{\mathbb{C}}$. As $\sigma$ and $\theta$ stabilize $\mathfrak{h}$, they induce actions on $\Delta$, defined by $\bar{\mu}(H):=\overline{\mu(H)}$ and $\theta \mu(H):=\mu(\theta(H))$, for $H \in \mathfrak{h}$, respectively. Fix a positive root system $\Delta^{+}$compatible with $\Sigma^{+}$, meaning that $\left.\mu\right|_{\mathfrak{a}}=\operatorname{Re}(\mu) \in \Sigma^{+}$ implies $\mu \in \Delta^{+}$. Then $\sigma \Delta^{+}=\Delta^{+}$.

Given a restricted root $\alpha \in \Sigma$, the corresponding restricted root space $\mathfrak{g}^{\alpha}$ decomposes into the direct sum of ordinary root spaces with respect to the Cartan subalgebra $\mathfrak{h}=\mathfrak{s} \oplus \mathfrak{a}$ as follows

$$
\mathfrak{g}^{\alpha}=\bigoplus_{\substack{\mu \in \Delta, \mu \neq \bar{\mu} \\ \operatorname{Re}(\mu)=\alpha}} \mathfrak{g}^{\mu} \oplus \mathfrak{g}^{\bar{\mu}} \oplus \mathfrak{g}^{\lambda}
$$

where $\lambda \in \Delta$ is possibly a root satisfying $\lambda=\bar{\lambda}$ and $\operatorname{Re}(\lambda)=\alpha$.

Lemma 2.2 (a) For $j=1, \ldots, r$, let $A_{j}$ and $P^{j}$ be as in (2) and (3). One has $I_{0} P^{j}=A_{j}$ and $I_{0} A_{j}=-P^{j}$. 
(b) Let $P=X-\theta X \in \mathfrak{p}\left[e_{j}+e_{l}\right]$, where $X=Z^{\mu}+\overline{Z^{\mu}} \in \mathfrak{g}^{e_{j}+e_{l}}$, with $Z^{\mu} \in \mathfrak{g}^{\mu}$, and $\mu \in \Delta^{+}$is a root satisfying $\operatorname{Re}(\mu)=e_{j}+e_{l}$ (if $\bar{\mu}=\mu$, we may assume $Z^{\mu}=\overline{Z^{\mu}}$ and set $\left.X=Z^{\mu}\right)$. Then $I_{0} P=Y-\theta Y$, where $Y=\left[K^{l}, X\right] \in \mathfrak{g}^{e_{j}-e_{l}}$.

(c) Let $P=X-\theta X \in \mathfrak{p}\left[e_{j}\right]$, where $X=Z^{\mu}+\overline{Z^{\mu}} \in \mathfrak{g}^{e_{j}}$, with $Z^{\mu} \in \mathfrak{g}^{\mu}$, and $\mu$ is a root in $\Delta^{+}$satisfying $\operatorname{Re}(\mu)=e_{j}\left(\right.$ as $\operatorname{dim} \mathfrak{p}\left[e_{j}\right]$ is even, one necessarily has $\left.\bar{\mu} \neq \mu\right)$. Then $I_{0} P=Y-\theta Y$, where $Y=i\left(Z^{\mu}-\overline{Z^{\mu}}\right) \in \mathfrak{g}^{e_{j}}$.

Proof (a) follows directly from (2) and (3).

Observe that $Z_{0} \in Z(\mathfrak{k})$ implies

$$
\left[Z_{0}, X\right]=-\left[Z_{0}, \theta X\right], \quad \text { for every } X \in \mathfrak{g} .
$$

(b) By (4), (5) and the fact that $\left[S_{0}, \mathfrak{g}^{\mu}\right] \subset \mathfrak{g}^{\mu}$, for every $\mu \in \Delta$, the action of $S_{0}$ is necessarily trivial on $\mathfrak{p}\left[e_{j}+e_{l}\right]$. Moreover, if $X \in \mathfrak{g}^{e_{j}+e_{l}}$, then $\left[K^{i}, X-\theta X\right]=0$, for all $i \neq j, l$, implying that

$$
\left[Z_{0}, X-\theta X\right]=\left[\frac{1}{2}\left(K^{j}+K^{l}\right), X-\theta X\right] .
$$

Denote by $\lambda$ the root in $\Delta$ with real part $e_{j}-e_{l}$ and the same imaginary part as $\mu$. By comparing terms in the same root spaces in (6), one obtains the relations

$$
\begin{aligned}
{\left[K^{l}, Z^{\mu}\right] } & =-\left[K^{j}, \theta Z^{\mu}\right] \in \mathfrak{g}^{\lambda} \quad\left[K^{l}, \overline{Z^{\mu}}\right]=-\left[K^{j}, \theta \overline{Z^{\mu}}\right] \in \mathfrak{g}^{\bar{\lambda}}, \\
{\left[K^{j}, Z^{\mu}\right] } & =-\left[K^{l}, \theta Z^{\mu}\right] \in \mathfrak{g}^{\theta \lambda} \quad\left[K^{j}, \overline{Z^{\mu}}\right]=-\left[K^{l}, \theta \overline{Z^{\mu}}\right] \in \mathfrak{g}^{\theta \bar{\lambda}} .
\end{aligned}
$$

It follows that $\left[Z_{0}, X-\theta X\right]=Y-\theta Y$, with $Y=\left[K^{l}, X\right] \in \mathfrak{g}^{e_{j}-e_{l}}$, as claimed.

(c) If $X \in \mathfrak{g}^{e_{j}}$, then $\left[K^{l}, X-\theta X\right]=0$, for all $l \neq j$, implying that $\left[Z_{0}, X-\theta X\right]=$ $\left[\frac{1}{2} K^{j}+S_{0}, X-\theta X\right]$. From (6) it follows

$$
\frac{1}{2}\left[K^{j}, X\right]+\left[S_{0}, X\right]=-\frac{1}{2}\left[K^{j}, \theta X\right]-\left[S_{0}, \theta X\right] .
$$

By comparing terms in the same root spaces, one obtains the relations

$$
\begin{array}{ll}
{\left[S_{0}, Z^{\mu}\right]=-\frac{1}{2}\left[K^{j}, \theta Z^{\mu}\right] \in \mathfrak{g}^{\mu}} & {\left[S_{0}, \theta Z^{\mu}\right]=-\frac{1}{2}\left[K^{j}, Z^{\mu}\right] \in \mathfrak{g}^{\theta \mu}} \\
{\left[S_{0}, \overline{Z^{\mu}}\right]=-\frac{1}{2}\left[K^{j}, \theta \overline{Z^{\mu}}\right] \in \mathfrak{g}^{\bar{\mu}}} & {\left[S_{0}, \theta \overline{Z^{\mu}}\right]=-\frac{1}{2}\left[K^{j}, \overline{Z^{\mu}}\right] \in \mathfrak{g}^{\theta \bar{\mu}},}
\end{array}
$$

which imply

$$
\left[Z_{0}, X-\theta X\right]=2\left(-\left[S_{0}, \theta Z^{\mu}\right]-\left[S_{0}, \theta \overline{Z^{\mu}}\right]+\left[S_{0}, Z^{\mu}\right]+\left[S_{0}, \overline{Z^{\mu}}\right]\right) .
$$

As $\mu\left(S_{0}\right)=: i \mu_{0} \in i \mathbb{R}$, the above expression becomes

$$
2 \mu_{0} i\left(Z^{\mu}-\overline{Z^{\mu}}-\theta\left(Z^{\mu}-\overline{Z^{\mu}}\right)\right) .
$$

From $I_{0}^{2}=-I d$, one obtains $\mu_{0}= \pm \frac{1}{2}$. Depending on the value $\mu_{0}$, the pairs of roots $\mu, \bar{\mu}$ can be relabelled so that $I_{0} P$ has the desired expression.

Remark 2.3 In view of Lemma 2.2, one can choose a $I_{0}$-stable basis of $\mathfrak{p}$, compatible with the decomposition (1).

(a) As a basis of $\mathfrak{a} \oplus \bigoplus_{j} \mathfrak{p}\left[2 e_{j}\right]$, take pairs of elements $A_{j}, P^{j}=-I_{0} A_{j}$, for $j=1, \ldots, r$, normalized as in (2) and (3); 
(b) As a basis of $\mathfrak{p}\left[e_{j}+e_{l}\right] \oplus \mathfrak{p}\left[e_{j}-e_{l}\right]$, take 4-tuples of elements $P, P^{\prime}, I_{0} P, I_{0} P^{\prime}$, parametrized by the pairs of roots $\mu \neq \bar{\mu} \in \Delta^{+}$satisfying $\operatorname{Re}(\mu)=e_{j}+e_{l}$ (with no repetition). More precisely, one has

$$
P=X-\theta X, \quad P^{\prime}=X^{\prime}-\theta X^{\prime}, \quad I_{0} P=Y-\theta Y, \quad I_{0} P^{\prime}=Y^{\prime}-\theta Y^{\prime},
$$

where $X=Z^{\mu}+\overline{Z^{\mu}}, X^{\prime}=i\left(Z^{\mu}-\overline{Z^{\mu}}\right), Y=\left[K^{l}, X\right]$ and $Y^{\prime}=\left[K^{l}, X^{\prime}\right]$, with $Z^{\mu}$ a root vector in $\mathfrak{g}^{\mu}$. For $\mu=\bar{\mu}$, one may assume $Z^{\mu}=\overline{Z^{\mu}}$ and take the pair $P, I_{0} P$.

(c) As a basis of $\mathfrak{p}\left[e_{j}\right]$ (non-tube case), take pairs of elements $P, I_{0} P$, parametrized by the pairs of roots $\mu \neq \bar{\mu} \in \Delta^{+}$satisfying $\operatorname{Re}(\mu)=e_{j}$ (with no repetition). More precisely, one has $P=X-\theta X$ and $I_{0} P=Y-\theta Y$, where $X=Z^{\mu}+\overline{Z^{\mu}}$, and $Y=i\left(Z^{\mu}-\overline{Z^{\mu}}\right)$, with $Z^{\mu}$ a root vector in $\mathfrak{g}^{\mu}$.

Lemma 2.4 Let $\mu \in \Delta^{+}$be a root satisfying $\operatorname{Re}(\mu)=e_{j}+e_{l}$ and let $Z^{\mu}$ be a root vector in $\mathfrak{g}^{\mu}$. Let $X=Z^{\mu}+\overline{Z^{\mu}} \in \mathfrak{g}^{e_{j}+e_{l}}$ and $Y=\left[K^{l}, X\right] \in \mathfrak{g}^{e_{j}-e_{l}}$. Then

(a) $[Y, X]+\theta[Y, X]=\left[Y^{\prime}, X^{\prime}\right]+\theta\left[Y^{\prime}, X^{\prime}\right]=r K^{j}$, for some $r \in \mathbb{R}$;

(b) $[Y, \theta X]+\theta[Y, \theta X]=\left[Y^{\prime}, \theta X^{\prime}\right]+\theta\left[Y^{\prime}, \theta X^{\prime}\right]=s K^{l}$, for some $s \in \mathbb{R}$.

If $\bar{\mu} \neq \mu$, let $X^{\prime}=i\left(Z^{\mu}-\overline{Z^{\mu}}\right)$ and $Y^{\prime}=\left[K^{l}, X^{\prime}\right]$. Then

(c) $\left[Y^{\prime}, X\right]+\theta\left[Y^{\prime}, X\right]=\left[Y^{\prime}, \theta X\right]+\theta\left[Y^{\prime}, \theta X\right]=0$.

Let $\mu$ be a root in $\Delta^{+}$, with $\operatorname{Re}(\mu)=e_{j}$ (non-tube case) and let $Z^{\mu}$ be a root vector in $\mathfrak{g}^{\mu}$. Let $X=Z^{\mu}+\overline{Z^{\mu}}$ and $Y=\left[Z_{0}, X\right]=i\left(Z^{\mu}-\overline{Z^{\mu}}\right)$. Then

(d) $[Y, X]+\theta[Y, X]=t K^{j}$, for some $t \in \mathbb{R}$,

(e) $[Y, \theta X]+\theta[Y, \theta X] \in \mathfrak{m}$.

Proof (a) One has $[Y, X]=\left[Y^{\prime}, X^{\prime}\right]=2 \operatorname{Re}\left[\left[\theta E^{l}, Z^{\mu}\right], \overline{Z^{\mu}}\right] \in \mathfrak{g}^{2 e_{j}}$. Since the root space $\mathfrak{g}^{2 e_{j}}$ is 1-dimensional, then

$$
[Y, X]+\theta[Y, X]=\left[Y^{\prime}, X^{\prime}\right]+\theta\left[Y^{\prime}, X^{\prime}\right]=r K^{j}, \quad \text { for some } r \in \mathbb{R} .
$$

(b) Similarly, $[Y, \theta X]=\left[Y^{\prime}, \theta X^{\prime}\right]=2 \operatorname{Re}\left[\left[\theta E^{l}, Z^{\mu}\right], \theta \overline{Z^{\mu}}\right] \in \mathfrak{g}^{-2 e_{l}}$, and

$$
[Y, \theta X]+\theta[Y, \theta X]=\left[Y^{\prime}, \theta X^{\prime}\right]+\theta\left[Y^{\prime}, \theta X^{\prime}\right]=s K^{l}, \quad \text { for some } s \in \mathbb{R} .
$$

(c) One has

$$
\begin{aligned}
{\left[Y^{\prime}, X\right] } & =\left[\left[K^{l}, i Z^{\mu}-i \overline{Z^{\mu}}\right], Z^{\mu}+\overline{Z^{\mu}}\right] \\
& =i\left[\left[K^{l}, Z^{\mu}\right], Z^{\mu}\right]+i\left[\left[K^{l}, Z^{\mu}\right], \overline{Z^{\mu}}\right]-i\left[\left[K^{l}, \overline{Z^{\mu}}\right], Z^{\mu}\right]-i\left[\left[K^{l}, \overline{Z^{\mu}}\right], \overline{Z^{\mu}}\right] .
\end{aligned}
$$

The first and the fourth terms of the above expression are both zero because otherwise there would exist a root in $\Delta^{+}$with real part equal to $2 e_{j}$ and non-zero imaginary part. The second and the third term sum up to zero by the Jacobi identity and the fact that $\left[K^{l},\left[Z^{\mu}, \overline{Z^{\mu}}\right]\right]=0$.

One has

$$
\begin{aligned}
{\left[Y^{\prime}, \theta X\right]=} & {\left[\left[K^{l}, i Z^{\mu}-i \overline{Z^{\mu}}\right], \theta Z^{\mu}+\theta \overline{Z^{\mu}}\right] } \\
= & i\left[\left[K^{l}, Z^{\mu}\right], \theta Z^{\mu}\right]+i\left[\left[K^{l}, Z^{\mu}\right], \theta \overline{Z^{\mu}}\right]-i\left[\left[K^{l}, \overline{Z^{\mu}}\right], \theta Z^{\mu}\right] \\
& -i\left[\left[K^{l}, \overline{Z^{\mu}}\right], \theta \overline{Z^{\mu}}\right] .
\end{aligned}
$$

Arguing as in the previous case, the first and the fourth terms are equal to zero. The second and the third terms sum up to $2 \operatorname{Im}\left(\left[\left[K^{l}, Z^{\mu}\right], \theta \overline{Z^{\mu}}\right]\right)$. Then

$$
\begin{aligned}
{\left[Y^{\prime}, \theta X\right]+\theta\left[Y^{\prime}, \theta X\right] } & =2 \operatorname{Im}\left(\left[\left[K^{l}, Z^{\mu}\right], \theta \overline{Z^{\mu}}\right]+\theta\left[\left[K^{l}, Z^{\mu}\right], \theta \overline{Z^{\mu}}\right]\right)= \\
& =2 \operatorname{Im}\left(\left[\left[K^{l}, Z^{\mu}\right], \theta \overline{Z^{\mu}}\right]+\left[\left[K^{l}, \theta Z^{\mu}\right], \overline{Z^{\mu}}\right]\right) .
\end{aligned}
$$


By the Jacobi identity

$$
\left[\left[K^{l}, \theta Z^{\mu}\right], \overline{Z^{\mu}}\right]=\left[\left[K^{l}, \overline{Z^{\mu}}\right], \theta Z^{\mu}\right]+\left[K^{l},\left[\theta Z^{\mu}, \overline{Z^{\mu}}\right]\right] .
$$

Observe that $\left[Z^{\mu}, \theta \overline{Z^{\mu}}\right] \in \mathfrak{a} \oplus i \mathfrak{s}$. Since $K^{l}$ centralizes $\mathfrak{s}$, one has that $\left[K^{l},\left[\theta Z^{\mu}, \overline{Z^{\mu}}\right]\right] \in$ $\mathfrak{p}$. It follows that the expression in (8) reduces to

$$
2 \operatorname{Im}\left(\left[\left[K^{l}, Z^{\mu}\right], \theta \overline{Z^{\mu}}\right]+\left[\left[K^{l}, \overline{Z^{\mu}}\right], \theta Z^{\mu}\right]\right)=0,
$$

as desired.

(d) Since $[Y, X] \in \mathfrak{g}^{2 e_{j}}$

$$
[Y, X]+\theta[Y, X]=t K^{j}, \quad \text { for some } t \in \mathbb{R} .
$$

(e) Since $[Y, \theta X] \in \mathfrak{g}^{0}$,

$$
[Y, \theta X]+\theta[Y, \theta X] \in \mathfrak{m}
$$

For $X \in \mathfrak{g}$, denote by $\widetilde{X}$ the vector field induced on $G / K$ by the left $G$-action, namely

$$
\tilde{X}_{z}:=\left.\frac{d}{d s}\right|_{s=0} \exp s X \cdot z
$$

for $z \in G / K$. Given a smooth $K$-invariant function $f: G / K \rightarrow \mathbb{R}$, define $d^{c} \rho:=d \rho \circ I_{0}$, where $I_{0}$ is the $G$-invariant complex structure of $G / K$. Then one has $2 i \partial \bar{\partial} f=-d d^{c} f$. For $X \in \mathfrak{k}$, consider the function $\mu^{X}: G / K \rightarrow \mathbb{R}$ given by $\mu^{X}(z):=d^{c} f\left(\widetilde{X}_{z}\right)$.

One has

$$
d \mu^{X}=-\iota \widetilde{X} d d^{c} f .
$$

The above identity was proved in [9], Lemma 7.1, for $f$ strictly plurisubharmonic. However the same argument works for arbitrary smooth $K$-invariant functions. This result will be used to compute the Levi form of an arbitrary smooth $K$-invariant function on $G / K$.

When the function $f$ is strictly plurisubharmonic, then $-d d^{c} f$ is a $K$-invariant Kähler form and the map $\mu: G / K \rightarrow \mathfrak{k}^{*}$, defined by

$$
\mu(z)(X)=d^{c} f\left(\widetilde{X}_{z}\right), \quad \text { for } X \in \mathfrak{k},
$$

is a moment map. It is referred to as the moment map associated with $f$.

We conclude the preliminaries with a lemma which is needed in the next section. Let $\Delta$ be the unit disc in $\mathbb{C}$. Consider the $\left(T \ltimes \mathcal{S}_{2}\right)$-action on the bidisk $\Delta^{2}$, where $T=\left(S^{1}\right)^{2}$ acts by rotations and $\mathcal{S}_{2}$ by permutations of the coordinates. Let $W_{\mathbb{R}^{2}}=\left(\mathbb{Z}_{2}\right)^{2} \ltimes \mathcal{S}_{2}$ be the group acting on $\mathbb{R}^{2}$ by signed permutations of the coordinates.

Lemma 2.5 Let $f: \Delta^{2} \rightarrow \mathbb{R}$ be a smooth $T \ltimes \mathcal{S}_{2}$-invariant strictly plurisubharmonic

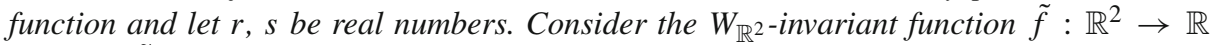
given by $\tilde{f}\left(a_{1}, a_{2}\right)=f\left(\tanh a_{1}, \tanh a_{2}\right)$ and define

$$
G_{\tilde{f}}\left(a_{1}, a_{2}\right):=\frac{r \sinh \left(2 a_{1}\right) \frac{\partial \widetilde{f}}{\partial a_{1}}\left(a_{1}, a_{2}\right)-s \sinh \left(2 a_{2}\right) \frac{\partial \widetilde{f}}{\partial a_{2}}\left(a_{1}, a_{2}\right)}{\sinh ^{2} a_{1}-\sinh ^{2} a_{2}} .
$$

Then

(i) $\frac{\partial \tilde{f}}{\partial a_{1}}\left(a_{1}, a_{2}\right)>0$, for every $a_{1}>0$, and $\frac{\partial \widetilde{f}}{\partial a_{1}}\left(a_{1}, a_{2}\right)<0$, for every $a_{1}<0$. In particular $\frac{\partial \tilde{f}}{\partial a_{1}}\left(0, a_{2}\right)=0$, for every $a_{2} \in \mathbb{R}$.

(ii) $\frac{\partial \widetilde{f}}{\partial a_{2}}\left(a_{1}, a_{2}\right)=\frac{\partial \tilde{f}}{\partial a_{1}}\left(a_{2}, a_{1}\right)$. In particular $\frac{\partial \tilde{f}}{\partial a_{2}}\left(a_{1}, 0\right)=0$, for every $a_{1} \in \mathbb{R}$. 
(iii) If $G_{\tilde{f}}$ extends continuously to a strictly positive function on $\mathbb{R}^{2}$, then $r=s>0$ and,

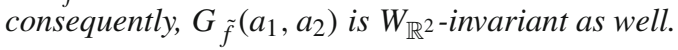

Proof (i) For $a_{1}>0$, write tanh $a_{1}=e^{s_{1}}$, for some $s_{1} \in(-\infty, 0)$. Since $f$ is $T$-invariant and strictly plurisubharmonic, the function $s_{1} \rightarrow f\left(e^{s_{1}}, \tanh a_{2}\right)$ is strictly convex and the limit $\lim _{s_{1} \rightarrow-\infty} f\left(e^{s_{1}}, \tanh a_{2}\right)=f\left(0, \tanh a_{2}\right)$ is finite. Hence the function is strictly increasing and its derivative $e^{s_{1}} \frac{\partial f}{\partial x_{1}}\left(e^{s_{1}}, \tanh a_{2}\right)$ is strictly positive. As

$$
\frac{\partial \tilde{f}}{\partial a_{1}}\left(a_{1}, a_{2}\right)=\frac{1}{\cosh ^{2} a_{1}} \frac{\partial f}{\partial x_{1}}\left(\tanh a_{1}, \tanh a_{2}\right),
$$

and $\tanh a_{1}=e^{s_{1}}$, the first part of statement (i) follows. The second one follows from the $\left(\mathbb{Z}_{2}\right)^{2}$-invariance of $\tilde{f}$.

(ii) The $\mathcal{S}_{2}$-invariance of $\tilde{f}$ implies that

$$
\lim _{\varepsilon \rightarrow 0} \frac{\tilde{f}\left(a_{1}, a_{2}+\varepsilon\right)}{\varepsilon}=\lim _{\varepsilon \rightarrow 0} \frac{\tilde{f}\left(a_{2}+\varepsilon, a_{1}\right)}{\varepsilon},
$$

and (ii) follows.

(iii) Let $a_{1}>0$. From (ii) it follows that $G_{\tilde{f}}\left(a_{1}, 0\right)=\frac{r \sinh \left(2 a_{1}\right) \frac{\partial \widetilde{f}}{\partial a_{1}}\left(a_{1}, 0\right)}{\sinh ^{2} a_{1}}$. Since such quantity is assumed to be strictly positive and $\frac{\partial \widetilde{f}}{\partial a_{1}}\left(a_{1}, 0\right)>0$, then $r>0$. By taking $a_{1}=0$ and $a_{2}>0$, one obtains that $s>0$.

Next we show that $r=s$. For $a_{1}>a_{2}>0$ one has $\sinh ^{2} a_{1}-\sinh ^{2} a_{2}>0$. Then the positivity of $G_{\tilde{f}}\left(a_{1}, a_{2}\right)$ implies that

$$
\frac{r}{s}>\frac{\sinh \left(2 a_{2}\right) \frac{\partial \widetilde{f}}{\partial a_{2}}\left(a_{1}, a_{2}\right)}{\sinh \left(2 a_{1}\right) \frac{\partial \tilde{f}}{\partial a_{1}}\left(a_{1}, a_{2}\right)} .
$$

Consequently, for $a_{1}$ converging to a fixed $a_{2}>0$, statements (i) and (ii) imply $\frac{r}{s} \geq$ 1. An analogous argument, with $0<a_{1}<a_{2}$, implies $\frac{r}{s} \leq 1$. As a consequence, $\frac{r}{s}=1$.

\section{The Levi form of a $K$-invariant function}

Let $G / K$ be an irreducible non-compact Hermitian symmetric space of rank $r$. From the decomposition $G=K \exp \mathfrak{a}$, every $K$-invariant domain $D$ in $G / K$ is uniquely determined by a $W$-invariant domain $\mathcal{D}_{\mathfrak{a}}$ in $\mathfrak{a}$ by

$$
D=K \exp \mathcal{D}_{\mathfrak{a}} K / K .
$$

Similarly, every $K$-invariant function $f: D \rightarrow \mathbb{R}$ is uniquely determined by the $W$-invariant function $\tilde{f}: \mathcal{D}_{\mathfrak{a}} \rightarrow \mathbb{R}$, given by

$$
\tilde{f}(H)=f(\exp (H) K) .
$$

The goal of this section is to express the real symmetric $I_{0}$-invariant bilinear form

$$
h_{f}(\cdot, \cdot):=-d d^{c} f\left(\cdot, I_{0} \cdot\right)
$$

of a smooth $K$-invariant function $f$ on a $K$-invariant domain $D \subset G / K$ in terms of the first and second derivatives of the function $\tilde{f}$ on $\mathcal{D}_{\mathfrak{a}}$. This will enable us to characterize smooth 
$K$-invariant strictly plurisubharmonic functions on a Stein $K$-invariant domain $D$ in $G / K$ by an appropriate differential positivity condition on the corresponding functions on $\mathcal{D}_{\mathfrak{a}}$ (see Theorem 4.5 and Corollary 4.6). As $f$ is $K$-invariant, $h_{f}$ is $K$-invariant as well. Therefore it will be sufficient to carry out the computations along the slice $\exp \mathcal{D}_{\mathfrak{a}} K$, which meets all the $K$-orbits in $D$.

For $z=a K$, with $a=\exp (H)$ and $H \in \mathfrak{a}$, one has

$$
\tilde{X}_{z}=a_{*} F_{a} X
$$

for all $X \in \mathfrak{g}$, where $F_{a}: \mathfrak{g} \rightarrow \mathfrak{p}$ is the map given by $F_{a}:=\pi_{\#} \circ \operatorname{Ad}_{a^{-1}}$, and $\pi_{\#}: \mathfrak{g} \rightarrow \mathfrak{p}$ is the linear projection along $\mathfrak{k}$. One can verify that

$$
\widetilde{K}_{z}=-a_{*} \sinh \alpha(H) P,
$$

for all $K=X^{\alpha}+\theta X^{\alpha} \in \mathfrak{k}[\alpha]$, with $\alpha \in \Sigma^{+}$and $P=X^{\alpha}-\theta X^{\alpha} \in \mathfrak{p}[\alpha]$.

Denote by $a_{1}, \ldots, a_{r}$ the coordinates induced on $\mathfrak{a}$ by the basis $A_{1}, \ldots, A_{r}$ of $\mathfrak{a}$ (cf. Remark 2.3(a)).

Proposition 3.1 Let $D \subset G / K$ be a $K$-invariant domain. Let $f: D \rightarrow \mathbb{R}$ be a smooth $K$-invariant function. Fix $a=\exp H$, with $H=\sum_{j} a_{j} A_{j} \in \mathcal{D}_{\mathfrak{a}}$. Then, in the basis of $\mathfrak{p}$ defined in Remark 2.3, the form $h_{f}$ at $z=a K \in D$ is given as follows.

(i) The spaces $a_{*} \mathfrak{a}, a_{*} I_{0} \mathfrak{a}, a_{*} \mathfrak{p}\left[e_{j}+e_{l}\right], a_{*} \mathfrak{p}\left[e_{j}-e_{l}\right]$ and $a_{*} \mathfrak{p}\left[e_{j}\right]$ are pairwise $h_{f}$ orthogonal.

As the form $h_{f}$ is $I_{0}$-invariant, by (5) it is determined by its restrictions to the blocks $a_{*} \mathfrak{a}$, $a_{*} \mathfrak{p}\left[e_{j}+e_{l}\right]$ and $a_{*} \mathfrak{p}\left[e_{j}\right]$. The non-zero entries of $h_{f}$ on each of these blocks are given as follows.

(ii) For $A_{j}, A_{l} \in \mathfrak{a}$ one has $h_{f}\left(a_{*} A_{j}, a_{*} A_{l}\right)=2 \operatorname{coth}\left(2 a_{j}\right) \frac{\partial \tilde{f}}{\partial a_{j}}(H) \delta_{j l}+\frac{\partial^{2} \tilde{f}}{\partial a_{j} \partial a_{l}}(H)$.

(iii) For $P, P^{\prime} \in \mathfrak{p}\left[e_{j}+e_{l}\right]$ as in Remark 2.3(b) one has

$$
\begin{aligned}
h_{f}\left(a_{*} P, a_{*} P\right) & =h_{f}\left(a_{*} P^{\prime}, a_{*} P^{\prime}\right) \\
& =\frac{B(P, P)}{\mathbf{b}} \frac{1}{\sinh ^{2}\left(a_{j}\right)-\sinh ^{2}\left(a_{l}\right)}\left(\sinh \left(2 a_{j}\right) \frac{\partial \tilde{f}}{\partial a_{j}}(H)-\sinh \left(2 a_{l}\right) \frac{\partial \tilde{f}}{\partial a_{l}}(H)\right),
\end{aligned}
$$

where $\mathbf{b}:=B\left(A_{1}, A_{1}\right)=\cdots=B\left(A_{r}, A_{r}\right)$. In particular, with respect to the basis of $a_{*} \mathfrak{p}\left[e_{j}+e_{l}\right]$ defined in Remark 2.3(b), the form $h_{f}$ is diagonal.

(iv) (non-tube case) For $P \in \mathfrak{p}\left[e_{j}\right]$ as in Remark 2.3 (c) one has

$$
h_{f}\left(a_{*} P, a_{*} P\right)=\frac{B(P, P)}{\mathbf{b}} \operatorname{coth}\left(a_{j}\right) \frac{\partial \tilde{f}}{\partial a_{j}}(H) .
$$

In particular, with respect to the basis of $a_{*} \mathfrak{p}\left[e_{j}\right]$ defined in Remark $2.3(c)$, the form $h_{f}$ is diagonal.

Proof We compute the form $h_{f}$ by exploiting relation (10). We begin by determining $d^{c} f\left(\tilde{X}_{z}\right)$, for $X \in \mathfrak{k}$ and $z \in G / K$. By the $K$-invariance of $f$ and of $I_{0}$ one has

$$
d^{c} f\left(\tilde{X}_{k \cdot z}\right)=d^{c} f\left(\widetilde{\mathrm{Ad}_{k^{-1}}} X_{z}\right),
$$

for every $z \in G / K$ and $k \in K$. Thus it is sufficient to take $z=a K$ in $\exp \mathcal{D}_{\mathfrak{a}} K$. We first assume that $\alpha(H) \neq 0$ for all $\alpha \in \Sigma$, and later obtain the complete result by passing to the limit for $H$ approaching the hyperplanes $\{\alpha=0\}$ in $\mathfrak{a}$. 
On the blocks of decomposition (1) of $\mathfrak{k}$, one has

$$
d^{c} f\left(\tilde{X}_{z}\right)=\left\{\begin{array}{cl}
-\sinh \left(2 a_{j}\right) \frac{\partial \tilde{f}}{\partial a_{j}}(H), & \text { for } X=K^{j} \\
0, & \text { for } X \in \mathfrak{k}[\alpha], \text { with } \alpha \neq 2 e_{1}, \ldots, 2 e_{r} .
\end{array}\right.
$$

Indeed, for $M \in \mathfrak{m}$, one has $\widetilde{M}_{z}=0$ and therefore $d^{c} f\left(\widetilde{M}_{z}\right)=0$.

Let $K=X+\theta X \in \mathfrak{k}[\alpha]$, for some $X \in \mathfrak{g}^{\alpha}$, with $\alpha \neq 2 e_{1}, \ldots, 2 e_{r}$. Set $P=X-$ $\theta X \in \mathfrak{p}[\alpha]$. Then $I_{0} P=Y-\theta Y \in \mathfrak{p}[\beta]$, for some $Y \in \mathfrak{g}^{\beta}$, with $\beta \in \Sigma^{+}$(cf.(5)). Set $C=Y+\theta Y \in \mathfrak{k}[\beta]$. Then, by (15) and the $K$-invariance of $f$ and of $I_{0}$, one has

$$
\begin{aligned}
d^{c} f\left(\widetilde{K}_{z}\right) & =-d f\left(I_{0} a_{*} \sinh \alpha(H) P\right)=-d f\left(a_{*} \sinh \alpha(H) I_{0} P\right) \\
& =d f\left(\frac{\sinh \alpha(H)}{\sinh \beta(H)} \widetilde{C}_{z}\right)=0 .
\end{aligned}
$$

Finally, let $K^{j} \in \mathfrak{k}\left[2 e_{j}\right]$, for $j=1, \ldots r$ (cf.(3)). One has

$$
\begin{aligned}
d^{c} f\left(\widetilde{K}_{z}^{j}\right) & =-d f\left(I_{0} a_{*} \sinh \left(2 a_{j}\right) P^{j}\right)=-d f\left(a_{*} \sinh \left(2 a_{j}\right) A_{j}\right) \\
& =-\left.\frac{d}{d s}\right|_{s=0} f\left(\exp \left(H+\sinh \left(2 a_{j}\right) s A_{j}\right) K\right)=-\sinh \left(2 a_{j}\right) \frac{\partial \widetilde{f}}{\partial a_{j}}(H) .
\end{aligned}
$$

Proof of statement (i). As a first step we show that $a_{*} \mathfrak{p}[\alpha]$ and $a_{*} \mathfrak{p}[\gamma]$ are $h_{f}$-orthogonal for any distinct roots $\alpha \in \Sigma^{+}$and $\gamma \in\{0\} \cup\left(\Sigma^{+} \backslash\left\{2 e_{1}, \ldots, 2 e_{r}\right\}\right)$, with the convention $\mathfrak{p}[0]:=\mathfrak{a}$.

Let $P \in \mathfrak{p}[\alpha]$ and $Q \in \mathfrak{p}[\gamma]$. Write $P=X-\theta X$, with $X \in \mathfrak{g}^{\alpha}$, and $I_{0} Q=Y-\theta Y$, with $Y \in \mathfrak{g}^{\beta}$, for some $\beta \in \Sigma^{+}$(cf.(5)). Then by (15) we have

$$
a_{*} P=-\frac{1}{\sinh \alpha(H)} \widetilde{K}_{z} \quad \text { and } \quad a_{*} I_{0} Q=-\frac{1}{\sinh \beta(H)} \widetilde{C}_{z},
$$

for $K=X+\theta X \in \mathfrak{k}[\alpha]$ and $C=Y+\theta Y \in \mathfrak{k}[\beta]$, respectively. Therefore

$$
\begin{aligned}
& h_{f}\left(a_{*} P, a_{*} Q\right)=-d d^{c} f\left(a_{*} P, a_{*} I_{0} Q\right)=\left.\frac{1}{\sinh \alpha(H) \sinh \beta(H)} \frac{d}{d t}\right|_{t=0} \mu^{K}(\exp t C \cdot z) \\
&= \\
&\left.\frac{1}{\sinh \alpha(H) \sinh \beta(H)} \frac{d}{d t}\right|_{t=0} d^{c} f\left(\widetilde{K}_{\exp t C \cdot z}\right)
\end{aligned}
$$

which, by (16), becomes

$$
\begin{aligned}
h_{f}\left(a_{*} P, a_{*} Q\right) & =\left.\quad \frac{1}{\sinh \alpha(H) \sinh \beta(H)} \frac{d}{d t}\right|_{t=0} d^{c} f\left(\widetilde{\operatorname{Ad}_{\exp -t C}} K_{z}\right) \\
& \left.=\left.\frac{1}{\sinh \alpha(H) \sinh \beta(H)} \frac{d}{d t}\right|_{t=0} d^{c} f\left(\widetilde{K}_{z}-t \widetilde{[C, K}\right]_{z}+o\left(t^{2}\right)\right) \\
& =\quad-\frac{1}{\sinh \alpha(H) \sinh \beta(H)} d^{c} f\left(\widetilde{[C, K]_{z}}\right) .
\end{aligned}
$$

The brackets

$$
[C, K]=([Y, X]+\theta[Y, X])+([Y, \theta X]+\theta[Y, \theta X]),
$$

lie in $\mathfrak{k}[\alpha+\beta]+\mathfrak{k}[\alpha-\beta]$. Since $\alpha \in \Sigma^{+}$and $\gamma \in\{0\} \cup\left(\Sigma^{+} \backslash\left\{2 e_{1}, \ldots, 2 e_{r}\right\}\right)$ are distinct, the spaces $\mathfrak{k}[\alpha+\beta]$ and $\mathfrak{k}[\alpha-\beta]$ have trivial intersection with $\oplus_{j} \mathfrak{k}\left[2 e_{j}\right]$. Then the expression (18) vanishes by (17), i.e. the spaces $a_{*} \mathfrak{p}[\alpha]$ and $a_{*} \mathfrak{p}[\gamma]$ are $h_{f}$-orthogonal. By the $I_{0}$ invariance of $h_{f}$, also $a_{*} I_{0} \mathfrak{a}$ is $h_{f}$-orthogonal to $a_{*} I_{0} \mathfrak{p}[\alpha]$, for all $\alpha \in \Sigma^{+}$. This concludes the proof of (i).

Next we examine the form $h_{f}$ on the blocks $a_{*} \mathfrak{a}, a_{*} \mathfrak{p}\left[e_{j}+e_{l}\right]$ and $a_{*} \mathfrak{p}\left[e_{j}\right]$.

(ii) The form $h_{f}$ on $a_{*}$ a. 
Let $A_{j}, A_{l} \in \mathfrak{a}$. Since $I_{0} A_{l}=-P^{l}$, one has

$$
\begin{aligned}
h_{f}\left(a_{*} A_{j}, a_{*} A_{l}\right) & =\quad-d d^{c} f\left(a_{*} P^{l}, a_{*} A_{j}\right)=\frac{1}{\sinh \left(2 a_{l}\right)} d d^{c} f\left(\left(\widetilde{K^{l}}\right)_{z},\left(\widetilde{A_{j}}\right)_{z}\right) \\
& =-\left.\frac{1}{\sinh \left(2 a_{l}\right)} \frac{d}{d t}\right|_{t=0} \mu^{K^{l}}\left(\exp t A_{j} \cdot z\right)=-\left.\frac{1}{\sinh \left(2 a_{l}\right)} \frac{d}{d t}\right|_{t=0} d^{c} f\left(\widetilde{K}^{l} \exp t A_{j} \cdot z\right) \\
& =\left.\quad \frac{1}{\sinh \left(2 a_{l}\right)} \frac{d}{d t}\right|_{t=0} d f\left(I_{0}\left(\exp \left(H+t A_{j}\right)\right)_{*} \sinh 2 e_{l}\left(H+t A_{j}\right) P^{l}\right. \\
& =\left.\quad \frac{1}{\sinh \left(2 a_{l}\right)} \frac{d}{d t}\right|_{t=0} \sinh 2 e_{l}\left(H+t A_{j}\right) \frac{\partial \widetilde{f}}{\partial a_{l}}\left(H+t A_{j}\right) \\
& =\quad \frac{1}{\sinh \left(2 a_{l}\right)}\left(2 \cosh \left(2 a_{l}\right) \frac{\partial \widetilde{f}}{\partial a_{l}}(H) \delta_{j, l}+\sinh \left(2 a_{l}\right) \frac{\partial^{2} \widetilde{f}}{\partial a_{j} \partial a_{l}}(H)\right) .
\end{aligned}
$$

The above expression is well defined also for those $H=\sum_{j} a_{j} A_{j}$ with some zero coordinate. Assume for example $a_{l}=0$. As it is $W$-invariant, $\tilde{f}$ is an even function of the coordinate $a_{l}$. Consequently its derivative $\frac{\partial \widetilde{f}}{\partial a_{l}}$ vanishes for $a_{l}=0$ and

$$
\lim _{a_{l} \rightarrow 0} 2 \operatorname{coth}\left(2 a_{l}\right) \frac{\partial \tilde{f}}{\partial a_{l}}=\frac{\partial^{2} \tilde{f}}{\partial a_{l}^{2}}
$$

smoothly extends to the hyperplane $a_{l}=0$. This concludes the proof of (ii).

(iii) The form $h_{f}$ on $a_{*} \mathfrak{p}\left[e_{j}+e_{l}\right]$.

Let $P, Q \in \mathfrak{p}\left[e_{j}+e_{l}\right]$ be elements of the basis of Remark 2.3(b), arising from roots $\mu, v \in \Delta^{+}$, respectively, with $v \neq \mu, \bar{\mu}$. Then $h_{f}\left(a_{*} P, a_{*} Q\right)=0$, because $\left[Z^{\mu} \pm \overline{Z^{\mu}}, Z^{v} \pm\right.$ $\left.\overline{Z^{v}}\right]=0$, for all $Z^{\mu} \in \mathfrak{g}^{\mu}$ and $Z^{v} \in \mathfrak{g}^{v}$.

Next, let $P, P^{\prime} \in \mathfrak{p}\left[e_{j}+e_{l}\right]$ and $I_{0} P, I_{0} P^{\prime} \in \mathfrak{p}\left[e_{j}-e_{l}\right]$ be elements of the basis of Remark 2.3(b), arising from the same root $\mu \in \Delta^{+}$.

From (18) it follows that

$$
\left.h_{f}\left(a_{*} P, a_{*} P\right)=-\frac{1}{\sinh \left(a_{j}+a_{l}\right) \sinh \left(a_{j}-a_{l}\right)}\left(d^{c} f(\widetilde{[C, K}]_{z}\right)\right)
$$

where $K=X+\theta X$ and $C=Y+\theta Y$, for $X$ and $Y$ as in (7). By Lemma 2.4(a)(b) and (17), the above expression equals

$$
\begin{aligned}
& -\frac{1}{\sinh ^{2}\left(a_{j}\right)-\sinh ^{2}\left(a_{l}\right)}\left(r d^{c} f\left(\widetilde{K}^{j}\right)-s d^{c} f\left(\widetilde{K}_{z}^{l}\right)\right) \\
& \quad=\frac{1}{\sinh ^{2}\left(a_{j}\right)-\sinh ^{2}\left(a_{l}\right)}\left(r \sinh \left(2 a_{j}\right) \frac{\partial \tilde{f}}{\partial a_{j}}(H)-s \sinh \left(2 a_{l}\right) \frac{\partial \tilde{f}}{\partial a_{l}}(H)\right),
\end{aligned}
$$

for some $r, s \in \mathbb{R}$. In a similar way, one obtains

$$
h_{f}\left(a_{*} P^{\prime}, a_{*} P^{\prime}\right)=h_{f}\left(a_{*} P, a_{*} P\right),
$$

and, from Lemma 2.4(c),

$$
h_{f}\left(a_{*} P, a_{*} P^{\prime}\right)=0 .
$$

Also, by (i), one has $h_{f}\left(a_{*} P, a_{*} I_{0} P\right)=h_{f}\left(a_{*} P, a_{*} I_{0} P^{\prime}\right)=0$.

For the strictly plurisubharmonic potential $\rho$ of the Killing metric of $G / K$ given in Proposition 5.1 , the quantity in (19) smoothly extends to a strictly positive function on $\mathbb{R}^{2}$. Hence (iii) of Lemma 2.5 implies that $r=s>0$. Finally, as $h_{\rho}\left(a_{*} P, a_{*} P\right)=B(P, P)$, a simple computation shows that $r=B(P, P) / \mathbf{b}$. This concludes the proof of (iii).

(iv) The form $h_{f}$ on $a_{*} \mathfrak{p}\left[e_{j}\right]$.

Let $P, Q \in \mathfrak{p}\left[e_{j}\right]$ be elements of the basis of Remark 2.3 (c), arising from roots $\mu, v \in$ $\Delta^{+}$, respectively, with $v \neq \mu, \bar{\mu}$. Then $h_{f}\left(a_{*} P, a_{*} Q\right)=0$, because $\left[Z^{\mu} \pm \overline{Z^{\mu}}, Z^{v} \pm\right.$ 
$\left.\overline{Z^{v}}\right]=0$, for all $Z^{\mu} \in \mathfrak{g}^{\mu}$ and $Z^{v} \in \mathfrak{g}^{v}$. In addition, by the $I_{0}$-invariance of $h_{f}$ one has $h_{f}\left(a_{*} P, a_{*} I_{0} P\right)=0$.

In order to compute $h_{f}\left(a_{*} P, a_{*} P\right)$, write $P=X-\theta X$ and $I_{0} P=Y-\theta Y$, with $X=Z^{\mu}+\overline{Z^{\mu}}$ and $Y=i\left(Z^{\mu}-\overline{Z^{\mu}}\right)$ (Lemma 2.2(c)). Then, from (18) it follows that

$$
\left.h_{f}\left(a_{*} P, a_{*} P\right)=-\frac{1}{\sinh ^{2}\left(a_{j}\right)} d^{c} f(\widetilde{[C, K}]_{z}\right),
$$

for $K=X+\theta X$ and $C=Y+\theta Y$. By Lemma 2.4(d)(e), one obtains

$$
h_{f}\left(a_{*} P, a_{*} P\right)=-\frac{1}{\sinh ^{2}\left(a_{j}\right)} d^{c} f\left(t \widetilde{K}^{j} z\right)=2 t \operatorname{coth}\left(a_{j}\right) \frac{\partial \widetilde{f}}{\partial a_{j}}(H), \text { for some } t \in \mathbb{R} \text {. }
$$

The above formula smoothly extends to $\mathcal{D}_{\mathfrak{a}}$, since $\frac{\partial \tilde{f}}{\partial a_{j}}$ is identically zero on the hyperplane $a_{j}=0$.

Finally, by computing the above quantity for the strictly plurisubharmonic potential $\rho$ of the Killing metric of $G / K$ given in Proposition 5.1, one obtains that $t=B(P, P) / \mathbf{b}$. This completes the proof of statement (iv) and of the proposition.

Remark 3.2 The Levi form $L_{f}^{\mathbb{C}}$ of $f$ is given by

$$
L_{f}^{\mathbb{C}}(Z, \bar{W})=2\left(h_{f}(X, Y)+i h_{f}\left(X, I_{0} Y\right)\right),
$$

where $Z=X-i I_{0} X$ and $W=Y-i I_{0} Y$ are elements in $\left(\mathfrak{p}^{\mathbb{C}}\right)^{1,0}$. One easily sees that $L_{f}^{\mathbb{C}}$ is (strictly) positive definite if and only if $h_{f}$ is (strictly) positive definite.

\section{$4 K$-invariant psh functions vs. $W$-invariant logcvx functions}

Let $G / K$ be an irreducible non-compact Hermitian symmetric space of rank $r$ and let $D \subset$ $G / K$ be a Stein, $K$-invariant domain. The goal of this section is to prove a characterization of various classes of $K$-invariant plurisubharmonic functions on $D$ by appropriate conditions on the corresponding functions on $\mathcal{D}_{\mathfrak{a}}$ (see (12) and (13)). In the smooth case we prove that a smooth $K$-invariant function $f$ of $D$ is strictly plurisubharmonic if and only if the associated function $\tilde{f}$ satisfies a positivity condition arising from Proposition 3.1(ii).

As an application, in Corollary 4.8, we reproduce the characterization of Stein $K$-invariant domains in $G / K$ outlined in [2], Thm. $3^{\prime}$ and Thm. 4.

Denote by $\Delta^{r}$ the orbit of the base point $e K \in G / K$ under the product of the $r$ commuting copies of $S U(1,1)$ determined by (2) and (3). One has $\Delta^{r}=T \exp \mathfrak{a} K$, where $T \cong\left(S^{1}\right)^{r}$ is the $r$-dimensional torus in $K$ whose Lie algebra is generated by $K^{1}, \ldots, K^{r}$. It is wellknown that $\Delta^{r}$ may be identified with the unit polydisk in $\mathbb{C}^{r}$ (cf. [13], p.280), and under this identification

$$
\exp \left(a_{1}, \ldots, a_{r}\right) K=\left(\tanh \left(a_{1}\right), \ldots, \tanh \left(a_{1}\right)\right), \quad \text { for }\left(a_{1}, \ldots, a_{r}\right) \in \mathfrak{a} .
$$

The polydisk $\Delta^{r}$ is a "thick slice" for the $K$-action in $G / K$, in the sense that $G / K=K \cdot \Delta^{r}$. If $D$ is a $K$-invariant domain in $G / K$, then

$$
R:=D \cap \Delta^{r}
$$

is by definition the Reinhardt domain associated to $D$ and satisfies $D=K \cdot R$.

We will show that if $D$ is Stein, then $R$ is necessarily connected. It should be remarked that, despite its appellation, a Reinhardt domain is open in $\mathbb{C}^{r}$ but need not be connected (in our context the quotient of $R$ under the action induced by the Weyl group is always connected). 
For a Reinhardt domain $R$ in $\Delta^{r}$, define the set

$$
\mathcal{D}=\left\{\left(a_{1}, \ldots, a_{r}\right) \in \mathbb{R}^{r}:\left(\tanh a_{1}, \ldots, \tanh a_{r}\right) \in R\right\},
$$

and note that the image of the map

$$
\mathcal{D} \rightarrow R \quad\left(a_{1}, \ldots, a_{r}\right) \rightarrow\left(\tanh a_{1}, \ldots, \tanh a_{r}\right)
$$

coincides with $R \cap \mathbb{R}^{r}$. One has $R=T \cdot\left(R \cap \mathbb{R}^{r}\right)$, with $T \cong\left(S^{1}\right)^{r}$. Given a smooth $T$-invariant function $f: R \rightarrow \mathbb{R}$, define $\tilde{f}: \mathcal{D} \rightarrow \mathbb{R}$ by

$$
\tilde{f}\left(a_{1}, \ldots, a_{r}\right)=f\left(\tanh a_{1}, \ldots, \tanh a_{r}\right) .
$$

As $f$ is $T$-invariant, the function $\tilde{f}$ is $\left(\mathbb{Z}^{2}\right)^{r}$-invariant.

Denote by $\log \operatorname{Con} v^{\infty,+}(\mathcal{D})^{\left(\mathbb{Z}_{2}\right)^{r}}$ the class of smooth functions on $\mathcal{D}$ which are even in each variable and such that the form defined in (ii) of Proposition 3.1, i.e.

$$
2 \operatorname{coth}\left(2 a_{j}\right) \frac{\partial \tilde{f}}{\partial a_{j}}(H) \delta_{j l}+\frac{\partial^{2} \tilde{f}}{\partial a_{j} \partial a_{l}}(H)
$$

for $j, l=1, \ldots, r$, is strictly positive definite, for every $H \in \mathcal{D}$. The next proposition characterizes $T$-invariant smooth strictly plurisubharmonic functions on $R$ by elements in $\log \operatorname{Conv}^{\infty,+}(\mathcal{D})^{\left(\mathbb{Z}_{2}\right)^{r}}$. It is an intermediate step in the proof of the main theorem in the smooth case, but it may be of independent interest in the context of Reinhardt domains.

Proposition 4.1 Let $f$ be a smooth T-invariant function on a Reinhardt domain $R$ in $\Delta^{r}$. Then $f$ is strictly plurisubharmonic if and only if $\tilde{f}$ belongs to $\log \operatorname{Conv} v^{\infty,+}(\mathcal{D})^{\left(\mathbb{Z}_{2}\right)^{r}}$.

Proof In polar coordinates $\left(\rho_{j}, \theta_{j}\right)$, with $z_{j}=\rho_{j} e^{i \theta_{j}} \neq 0$, one has

$$
\partial_{z_{j}}=\frac{e^{-i \theta_{j}}}{2 \rho_{j}}\left(\rho_{j} \partial_{\rho_{j}}-i \partial_{\theta_{j}}\right) \quad \partial_{\bar{z}_{j}}=\frac{e^{i \theta_{j}}}{2 \rho_{j}}\left(\rho_{j} \partial_{\rho_{j}}+i \partial_{\theta_{j}}\right) .
$$

One easily sees that, for $z_{j} z_{l} \neq 0$,

$$
4 \frac{\partial^{2} f}{\partial \bar{z}_{j} \partial z_{l}}\left(z_{1}, \ldots, z_{r}\right)=\frac{1}{\rho_{j}} \frac{\partial f}{\partial \rho_{j}}\left(\rho_{1}, \ldots, \rho_{r}\right) \delta_{j l}+e^{i\left(\theta_{j}-\theta_{l}\right)} \frac{\partial^{2} f}{\partial \rho_{j} \partial \rho_{l}}\left(\rho_{1}, \ldots, \rho_{r}\right) .
$$

As it is $T$-invariant, $f$ is an even function in each of the variables $\rho_{1}, \ldots, \rho_{r}$. Consequently, the above quantity extends smoothly through the hyperplanes $z_{j}=0$ (and therefore to the whole domain) whenever $j=l$, while

$$
4 \frac{\partial^{2} f}{\partial \bar{z}_{j} \partial z_{l}}\left(z_{1}, \ldots, z_{r}\right)=0, \quad \text { for } j \neq l \text { and } z_{j} z_{l}=0 .
$$

For $\rho_{1}=\tanh a_{1}, \ldots, \rho_{r}=\tanh a_{r}$, one has

$$
\begin{aligned}
\frac{\partial \tilde{f}}{\partial a_{j}}\left(a_{1}, \ldots, a_{r}\right)= & \frac{\partial f}{\partial \rho_{j}}\left(\tanh a_{1}, \ldots, \tanh a_{r}\right) \frac{1}{\cosh ^{2} a_{j}}, \\
\frac{\partial^{2} \tilde{f}}{\partial a_{j} \partial a_{l}}(H)= & \frac{\partial^{2} f}{\partial \rho_{j} \partial \rho_{l}}\left(\tanh a_{1}, \ldots, \tanh a_{r}\right) \frac{1}{\cosh ^{2} a_{j} \cosh ^{2} a_{l}} \\
& -\delta_{j l} \frac{\partial f}{\partial \rho_{j}}\left(\tanh a_{1}, \ldots, \tanh a_{r}\right) \frac{2 \sinh a_{j}}{\cosh ^{3} a_{j}},
\end{aligned}
$$

and likewise

$$
\frac{\partial^{2} \tilde{f}}{\partial a_{j} \partial a_{l}}(H)=0, \quad \text { for } j \neq l \text { and } a_{j} a_{l}=0 .
$$


A simple computation combining formulas (23) and (24) with (22), shows that $4 \frac{\partial^{2} f}{\partial \bar{z}_{j} \partial z_{l}}\left(z_{1}, \ldots, z_{r}\right)$ is given by

$$
\begin{cases}\cosh ^{4} a_{j}\left(2 \operatorname{coth}\left(2 a_{j}\right) \frac{\partial \tilde{f}}{\partial a_{j}}\left(a_{1}, \ldots, a_{r}\right)+\frac{\partial^{2} \tilde{f}}{\partial a_{j}^{2}}\left(a_{1}, \ldots, a_{r}\right)\right), & \text { for } j=l, \\ \cosh ^{2} a_{j} e^{i \theta_{j}} \cosh ^{2} a_{l} e^{-i \theta_{l}} \frac{\partial^{2} \tilde{f}}{\partial a_{j} \partial a_{l}}\left(a_{1}, \ldots, a_{r}\right), & \text { for } j \neq l \text { and } z_{j} z_{l} \neq 0, \\ 0, & \text { for } j \neq l \text { and } z_{j} z_{l}=0 .\end{cases}
$$

Then, for $\left(z_{1}, \ldots, z_{r}\right) \in R$, one has

$$
\left(4 \frac{\partial^{2} f}{\partial \bar{z}_{j} \partial z_{l}}\right)_{j, l}=C\left(\frac{\partial^{2} \tilde{f}}{\partial a_{j} \partial a_{l}}+\delta_{j l} 2 \operatorname{coth}\left(2 a_{j}\right) \frac{\partial \tilde{f}}{\partial a_{j}}\right)_{j, l} \bar{C}
$$

where $C$ is the diagonal matrix with diagonal entries

$$
c_{j j}= \begin{cases}\cosh ^{2}\left(a_{j}\right) e^{i \theta_{j}}, & \text { for } z_{j} \neq 0, \\ \cosh ^{2}\left(a_{j}\right), & \text { for } z_{j}=0 .\end{cases}
$$

It follows that $f$ is strictly plurisubharmonic if and only if $\tilde{f}$ belongs to the class $\log \operatorname{Conv}^{\infty,+}(\mathcal{D})^{\left(\mathbb{Z}_{2}\right)^{r}}$.

Let $R$ be a Reinhardt domain in $\left(\Delta^{*}\right)^{r}$ and let

$$
\mathcal{D}_{\log }:=\left\{\left(s_{1}, \ldots, s_{r}\right) \in\left(\mathbb{R}^{<0}\right)^{r}:\left(e^{s_{1}}, \ldots, e^{s_{r}}\right) \in R\right\}
$$

be the logarithmic image of its slice $R \cap\left(\mathbb{R}^{>0}\right)^{r}$. For a $T$-invariant function $f$ on $R$, define $\widehat{f}: \mathcal{D}_{\log } \rightarrow \mathbb{R}$ by

$$
\widehat{f}\left(s_{1}, \ldots, s_{r}\right):=f\left(e^{s_{1}}, \ldots, e^{s_{r}}\right) .
$$

It is well known that if $f$ is smooth, then it is strictly plurisubharmonic if and only if $\widehat{f}$ has strictly positive definite Hessian. The next remarks elucidate the significance of the class $\log \operatorname{Conv}^{\infty,+}(\mathcal{D})^{\left(\mathbb{Z}_{2}\right)^{r}}$.

Remark 4.2 Let $R$ be a Reinhardt domain in $\left(\Delta^{*}\right)^{r}$ and let $f$ be a smooth $T$-invariant function on $R$. Then $\tilde{f}$ belongs to $\log \operatorname{Conv} v^{\infty,+}(\mathcal{D})^{\left(\mathbb{Z}_{2}\right)^{r}}$ if and only if the smooth function $\hat{f}$ has everywhere strictly positive Hessian.

Proof One has

$$
\begin{aligned}
& \frac{\partial \hat{f}}{\partial s_{j}}\left(s_{1}, \ldots, s_{r}\right)=\frac{\partial f}{\partial \rho_{j}}\left(e^{s_{1}}, \ldots, e^{s_{r}}\right) e^{s_{j}} \\
& \frac{\partial^{2} \hat{f}}{\partial s_{j} \partial s_{l}}\left(s_{1}, \ldots, s_{r}\right)=\frac{\partial^{2} f}{\partial \rho_{j} \partial \rho_{l}}\left(e^{s_{1}}, \ldots, e^{s_{r}}\right) e^{s_{j}} e^{s_{l}}+\delta_{j l} \frac{\partial f}{\partial \rho_{j}}\left(e^{s_{1}}, \ldots, e^{s_{r}}\right) e^{s_{j}} .
\end{aligned}
$$

Then, by letting $e^{s_{1}}=\tanh a_{1}, \ldots, e^{s_{r}}=\tanh a_{r}$, with $a_{1}, \ldots, a_{r}>0$, and combining formulas (26) and (27) with (23) and (24), one obtains

$$
2 \operatorname{coth}\left(2 a_{j}\right) \frac{\partial \tilde{f}}{\partial a_{j}}(H) \delta_{j l}+\frac{\partial^{2} \tilde{f}}{\partial a_{j} \partial a_{l}}(H)=\frac{4}{\sinh 2 a_{j} \sinh 2 a_{l}} \frac{\partial^{2} \hat{f}}{\partial s_{j} \partial s_{l}}\left(s_{1}, \ldots, s_{r}\right) .
$$

Hence $\tilde{f} \in \log \operatorname{Con} v^{\infty,+}(\mathcal{D})^{\left(\mathbb{Z}_{2}\right)^{r}}$ if and only if $\hat{f}$ has everywhere strictly positive Hessian.

Remark 4.3 Let $R$ be an arbitrary Reinhardt domain and let $\left.f\right|_{R \cap\left(\Delta^{*}\right)^{r}}$ denote the restriction of $f$ to $R \cap\left(\Delta^{*}\right)^{r}$. The strict positivity of the Hessian of $\left.f\right|_{R \cap\left(\Delta^{*}\right)^{r}}$ on $R \cap\left(\Delta^{*}\right)^{r}$ does not imply the strict plurisubharmonicity of $f$ on the coordinate hyperplanes (and therefore on 
the whole $R$ ). For instance, despite the fact that it has strictly positive Hessian on $R \cap \Delta^{*}$, the function $g(z)=|z|^{4}$ is not plurisubharmonic at $z=0$. In contrast, this fact is detected by the vanishing of the form

$$
\frac{\partial^{2} \widetilde{g}}{\partial a^{2}}(a)+2 \operatorname{coth}(2 a) \frac{\partial \widetilde{g}}{\partial a}(a)=16 \frac{\tanh ^{2} a}{\cosh ^{4} a}
$$

at $a=0$, which shows that the associated function $\tilde{g}(a)=\tanh ^{4}(a)$ does not belong to $\log \operatorname{Conv}^{\infty,+}(\mathbb{R})^{\left(\mathbb{Z}_{2}\right)}$.

Let $R \subset \Delta^{r}$ be a Reinhardt domain associated to a $K$-invariant domain in $G / K$. In this case, $R$ is also invariant under the coordinate permutations induced by the Weyl group action on $\mathfrak{a}$. If such a Reinhardt domain is Stein, then there are two possibilities:

(a) $R$ intersects the coordinate hyperplanes. Then it is complete (cf. [3], Thm.2.12). In particular, it contains the origin and it is connected.

(b) $R$ does not intersect the coordinate hyperplanes, i.e. $R \subset\left(\Delta^{*}\right)^{r}$. Then $R$ is logarithmically convex.

The next proposition shows that a Stein Reinhardt domain $R$ associated to a Stein $K$ invariant domain $D \subset G / K$ is necessarily connected (even in case (b), when $0 \notin R$ ), a fact already pointed out in $\left[2\right.$, Thm. $\left.3^{\prime}\right]$.

Proposition 4.4 Let $D$ and $R$ be as above and let $f: D \rightarrow \mathbb{R}$ be a smooth, $K$-invariant strictly plurisubharmonic exhaustion function of the Stein domain $D$.

(i) If $R$ contains the origin, then $R$ is connected and $\tilde{f}$ has a unique minimum point at the origin of $\mathcal{D}_{\mathfrak{a}}$.

(ii) If $R$ does not contain the origin, then $\tilde{f}$ has a unique minimum point on the intersection $\mathcal{D}_{\mathfrak{a}} \cap\left\{a_{1}=\cdots=a_{r}>0\right\}$. In particular $R$ is connected. In this case $G / K$ is necessarily of tube type.

Proof The minimum set of a $K$-invariant exhaustion function $f$ of $D$ intersects $R=T$. $\exp \mathcal{D}_{\mathfrak{a}} K$ in a non-empty $T$-invariant set. Moreover, $\exp (H) K \in R$ is a minimum point of $\left.f\right|_{R}$, the restriction of $f$ to $R$, if and only if $H \in \mathcal{D}_{\mathfrak{a}}$ is a minimum point of $\widetilde{f}$.

(i) As $R$ intersects the coordinate hyperplanes, it is complete, as mentioned above. Assume that $\tilde{f}$ has a minimum point $H=\left(a_{1}, \ldots, a_{r}\right)$, different from the origin. Then $\left.f\right|_{R}$ has a minimum point in $P=\exp (H) K$. For $\varepsilon>0$ small enough there is a holomorphic immersion

$$
\iota: \Delta_{1+\varepsilon} \rightarrow R, \quad z \rightarrow z P,
$$

where $\Delta_{1+\varepsilon}$ denotes the disc of center 0 and radius $1+\varepsilon$ in $\mathbb{C}$. The pull-back $f \circ \iota$ of $f$ via $\iota$ is a smooth strictly subharmonic $S^{1}$-invariant function on $\Delta_{1+\varepsilon}$. It has a minimum point in 0 and, by construction, in 1 . Then $f \circ \iota$ is necessarily constant, contradicting the fact that it is strictly subharmonic.

(ii) Let $H=\left(a_{1}, \ldots, a_{r}\right)$, with $a_{j} \geq 0$, be a minimum point of $\tilde{f}$. As $R$ does not intersect the coordinate hyperplanes, all $a_{j}$ 's are different from 0 . As a consequence $2 \operatorname{coth}\left(a_{j}\right) \frac{\partial \tilde{f}}{\partial a_{j}}(H)=0$, for $j=1, \ldots, r$. In the non-tube case this contradicts the strict plurisubharmonicity of $f$ by (iv) of Proposition 3.1, implying that the space $G / K$ is necessarily of tube type. The strict plurisubharmonicity of $f$ along with (iii) of Proposition 3.1, implies that $a_{j}=a_{k}$ for every $j, k=1, \ldots, r$. Hence $H$ lies on the positive diagonal of $\mathfrak{a}$. Consider the Weyl chamber $\mathfrak{a}^{+}=\left\{a_{1} \geq a_{2} \geq \cdots \geq a_{r} \geq\right.$ 
$0\}$. Since $\mathcal{D}_{\mathfrak{a}} \cap \mathfrak{a}^{+}$is connected by the connectedness of $D$ and $H$ belongs to the boundary of every Weyl chamber in $\left\{\left(a_{1}, \ldots a_{r}\right) \in \mathfrak{a}: a_{j} \geq 0, j=1, \ldots, r\right\}$, it follows that $R \cap\left(\mathbb{R}^{>0}\right)^{r}$ is connected (as well as $\mathcal{D}_{\text {log }}$ ). Hence the Reinhardt domain $R=T \cdot\left(R \cap\left(\mathbb{R}^{>0}\right)^{r}\right)$ is connected. The uniqueness of the minimum point of $\widetilde{f}$ follows from standard arguments as in [1], or from the following direct argument.

The region $\mathcal{D}_{\log }$ is convex by the Steinness of $D$. By Remark 4.2, the associated function $\hat{f}$ has everywhere strictly positive definite Hessian. In particular its restriction to the diagonal $\mathcal{D}_{\log } \cap\left\{s_{1}=\cdots=s_{r}\right\}$ is a strictly convex exhaustion function. Consequently it has a unique minimum point, implying that $\tilde{f}$ has a unique minimum point on $\mathcal{D}_{\mathfrak{a}} \cap\left\{a_{1}=\cdots=a_{r}>0\right\}$.

Consider the following classes of functions:

- $C^{0}\left(\mathcal{D}_{\mathfrak{a}}\right)^{W}$ : continuous $W$-invariant functions on $\mathcal{D}_{\mathfrak{a}}$,

- $C^{\infty}\left(\mathcal{D}_{\mathfrak{a}}\right)^{W}$ : smooth $W$-invariant functions on $\mathcal{D}_{\mathfrak{a}}$,

- $C^{0}(D)^{K}$ : continuous $K$-invariant functions on $D$,

- $C^{\infty}(D)^{K}$ : smooth $K$-invariant functions on $D$.

Since the $K$-action on $D$ is proper and every $K$-orbit intersects the slice $\exp \mathcal{D}_{\mathfrak{a}} K$ in a $W$-orbit, the map $f \rightarrow \tilde{f}$ is a bijection from $C^{0}(D)^{K}$ onto $C^{0}\left(\mathcal{D}_{\mathfrak{a}}\right)^{W}$. By Theorem 4.1 in [6] (see also [4]) such a map is also a bijection from $C^{\infty}(D)^{K}$ onto $C^{\infty}\left(\mathcal{D}_{\mathfrak{a}}\right)^{W}$. Define:

- $\log \operatorname{Conv}^{\infty,+}\left(\mathcal{D}_{\mathfrak{a}}\right)^{W}$ : smooth, $W$-invariant functions on $\mathcal{D}_{\mathfrak{a}}$ such that the form (21) is strictly positive definite,

- $P^{\infty,+}(D)^{K}$ : smooth, $K$-invariant, strictly plurisubharmonic functions (i.e. with strictly positive definite Levi form) on $D$.

Our first result is the following theorem.

Theorem 4.5 Let $D$ be a Stein $K$-invariant domain in an irreducible non-compact Hermitian symmetric space $G / K$ of rank $r$. Then $f \in P^{\infty,+}(D)^{K}$ if and only if $\tilde{f} \in$ $\log \operatorname{Conv}^{\infty,+}\left(\mathcal{D}_{\mathfrak{a}}\right)^{W}$.

Proof By (ii) of Proposition 3.1, if $f$ is strictly plurisubharmonic on $D$, then $\tilde{f} \in$ $\log \operatorname{Conv}^{\infty,+}\left(\mathcal{D}_{\mathfrak{a}}\right)^{W}$.

Conversely, assume that $\tilde{f} \in \log \operatorname{Con} v^{\infty,+}\left(\mathcal{D}_{\mathfrak{a}}\right)^{W}$. We need to show that the terms in (iii) and (iv) of Proposition 3.1 are strictly positive (the ones in (iii) occurring only if $r>1$, the ones in (iv) occurring only in the non-tube case).

For the terms in (iii), without loss of generality, it is sufficient to consider the case $r=2$, and $H=\left(a_{1}, a_{2}\right) \in \mathfrak{a}^{+}$, with $a_{1} \geq a_{2} \geq 0$. Assume first $a_{1}>a_{2}>0$. Then $\left(\tanh a_{1}, \tanh a_{2}\right)=\left(e^{s_{1}}, e^{s_{2}}\right) \in R^{*}$, where $R$ is the Reinhardt domain associated to $D$. Let $d_{0}<0$ and $t_{0}>0$ be real numbers defined by $\left(s_{1}, s_{2}\right)=\left(d_{0}+t_{0}, d_{0}-t_{0}\right)$.

From now on, refer to the smooth functions with everywhere positive definite Hessian as SSC (smooth stably convex). By Remark 4.2, the function $\hat{f}$, which is invariant under coordinate permutations, is SSC. Therefore $g(t):=\hat{f}\left(d_{0}+t, d_{0}-t\right)$ is even and SSC. Consequently, for $t_{0}$ as above, the inequality

$$
g^{\prime}\left(t_{0}\right)=\frac{\partial f}{\partial \rho_{1}}\left(e^{d_{0}+t_{0}}, e^{d_{0}-t_{0}}\right) e^{d_{0}+t_{0}}-\frac{\partial f}{\partial \rho_{2}}\left(e^{d_{0}+t_{0}}, e^{d_{0}-t_{0}}\right) e^{d_{0}-t_{0}}>0,
$$

holds true. This, combined with formulas (23), implies

$$
g^{\prime}\left(t_{0}\right)=\frac{1}{2}\left(\sinh \left(2 a_{2}\right) \frac{\partial \tilde{f}}{\partial a_{2}}(H)-\sinh \left(2 a_{1}\right) \frac{\partial \tilde{f}}{\partial a_{1}}(H)\right)>0,
$$


giving the desired positivity.

Next consider $H=(a, a)$, with $a \neq 0$. Set $\tanh a=e^{d_{0}}$ and $\left(\tanh a_{1}, \tanh a_{2}\right)=$ $\left(e^{d_{0}+t}, e^{d_{0}-t}\right)$. Recall that $g^{\prime}(0)=0$. Then the corresponding term in (iii) of Proposition 3.1 is the limit

$$
\begin{aligned}
\frac{1}{2} \lim _{t \rightarrow 0} \frac{g^{\prime}(t)}{\sinh \left(a_{1}+a_{2}\right) \sinh \left(a_{1}-a_{2}\right)} & =\frac{1}{4} \lim _{t \rightarrow 0} \frac{g^{\prime}(t)}{t} \frac{\left(d_{0}+t\right)-\left(d_{0}-t\right)}{\sinh \left(a_{1}+a_{2}\right) \sinh \left(a_{1}-a_{2}\right)} \\
& =\frac{1}{4} \frac{g^{\prime \prime}(0)}{\sinh (2 a) \cosh (2 a)} \lim _{\left(a_{1}-a_{2}\right) \rightarrow 0} \frac{\log \tanh a_{1}-\log \tanh a_{2}}{a_{1}-a_{2}}=g^{\prime \prime}(0) c(a),
\end{aligned}
$$

which is positive since $c(a)$ is a positive real number and $g^{\prime \prime}(0)>0$ ( $g$ is even and SSC).

If $H=\left(a_{1}, 0\right) \in \mathcal{D}_{\mathfrak{a}}$, with $a_{1}>0$, then the Reinhardt domain $R$ associated to $D$ is necessarily complete and the term to be evaluated reduces to

$$
\frac{1}{\sinh ^{2} a_{1}} \sinh \left(2 a_{1}\right) \frac{\partial \widetilde{f}}{\partial a_{1}}\left(a_{1}, 0\right) \text {. }
$$

As

$$
2 \operatorname{coth}\left(2 a_{1}\right) \frac{\partial \tilde{f}}{\partial a_{1}}\left(a_{1}, 0\right)+\frac{\partial^{2} \tilde{f}}{\partial a_{1}^{2}}\left(a_{1}, 0\right)>0
$$

the function $s_{1} \rightarrow f\left(e^{s_{1}}, 0\right)$ is SSC (cf.Rem.4.2). Since $R$ is complete, then $\lim _{s_{1} \rightarrow-\infty}$ $f\left(e^{s_{1}}, 0\right)$ is finite. As a consequence $s_{1} \rightarrow f\left(e^{s_{1}}, 0\right)$ is strictly increasing and so is $a_{1} \rightarrow$ $\tilde{f}\left(a_{1}, 0\right)=f\left(\tanh a_{1}, 0\right)$. Hence $\frac{\partial \tilde{f}}{\partial a_{1}}\left(a_{1}, 0\right)$ is positive, as wished.

Finally, for $a_{1}=a_{2}=0$, the analytic extension of our term is given by

$$
2 \frac{\partial^{2} \tilde{f}}{\partial a_{1}^{2}}(0,0)=2 \frac{\partial^{2} \tilde{f}}{\partial a_{2}^{2}}(0,0),
$$

which is strictly positive by assumption.

We are left to examine the terms in (iv), which only appear in the non-tube case. The arguments are similar to the ones used in the previous case. By Proposition 4.4, the Reinhardt domain $R$ associated to $D$ is complete. Then $\lim _{s_{j} \rightarrow-\infty} \hat{f}\left(s_{1}, \ldots, s_{j}, \ldots s_{r}\right)$ is finite. Since $\hat{f}$ is SSC, the function $s_{j} \rightarrow \hat{f}\left(s_{1}, \ldots, s_{j}, \ldots s_{r}\right)$ is strictly increasing and so is $a_{j} \rightarrow$ $\tilde{f}\left(a_{1}, \ldots, a_{j}, \ldots a_{r}\right)$. Hence

$$
2 \operatorname{coth}\left(a_{j}\right) \frac{\partial \tilde{f}}{\partial a_{j}}\left(a_{1}, \ldots a_{r}\right)>0, \text { for } a_{j}>0 .
$$

The limit

$$
\lim _{a_{j} \rightarrow 0} 2 \operatorname{coth}\left(a_{j}\right) \frac{\partial \tilde{f}}{\partial a_{j}}\left(a_{1}, \ldots, a_{j}, \ldots, a_{r}\right)=2 \frac{\partial^{2} \tilde{f}}{\partial a_{j}^{2}}\left(a_{1}, \ldots, 0, \ldots, a_{r}\right)
$$

is strictly positive as well, by assumption. This completes the proof of the theorem.

Consider the $\left(T \ltimes \mathcal{S}_{r}\right)$-action on $\Delta^{r}$, where $\mathcal{S}_{r}$ denotes the group of coordinate permutations. From Proposition 4.1 one deduces the following corollary.

Corollary 4.6 Let $D$ be a Stein $K$-invariant domain in an irreducible non-compact Hermitian symmetric space $G / K$ and let $R$ be the associated Reinhardt domain. The map $\left.f \rightarrow f\right|_{R}$ is a bijection between $P^{\infty,+}(D)^{K}$ and $P^{\infty,+}(R)^{T \ltimes \mathcal{S}_{r}}$.

Remark 4.7 If $R$ does not contain the origin, then, by Remark 4.2, the condition $f \in$ $P^{\infty,+}(D)^{K}$ is also equivalent to requiring that the smooth invariant function $\hat{f}$ has strictly positive definite Hessian on $\mathcal{D}_{\text {log }}$. 
Corollary 4.8 (See [2, Thm. 3' and Thm.4]) Let $D$ be a Stein $K$-invariant domain in an irreducible non-compact Hermitian symmetric space $G / K$ and let $R$ be the associated Reinhardt domain. Then

(i) If $G / K$ is of tube type, then $D$ is Stein if and only if $R$ is Stein and connected.

(ii) If $G / K$ is not of tube type, then $D$ is Stein if and only if $R$ is Stein and complete. In particular $R$ contains the origin and is connected.

Proof By Proposition 4.4, if $D$ is Stein then the intersection $R=D \cap \Delta^{r}$ is Stein, connected and, in the non-tube case, complete. Conversely, let $R$ be a Stein, connected Reinhardt domain, invariant under coordinate permutations. In the non-tube case also assume $R$ to be complete. Let $f$ be a smooth, strictly plurisubharmonic exhaustion function of $R$. After an averaging process, $f$ may be assumed to be invariant with respect to $T$ and to the coordinate permutations. Proposition 4.1 implies that the associated function $\tilde{f}: \mathcal{D}_{\mathfrak{a}} \rightarrow \mathbb{R}$ belongs to $\log \operatorname{Con} v^{\infty,+}\left(\mathcal{D}_{\mathfrak{a}}\right)^{W}$. By Theorem 4.5, $\tilde{f}$ extends to a smooth, $K$-invariant, strictly plurisubharmonic exhaustion function of $D$. Hence $D$ is Stein.

Remark 4.9 The envelope of holomorphy $\widehat{D}$ of a $K$-invariant domain $D$ in $G / K$ is described, without proof, in terms of the associate Reinhardt domain $R$ in Theorem 5 of [2]:

if $G / K$ is of tube type, then $\widehat{D}=K \cdot \widetilde{R}$, where $\widetilde{R}$ is the smallest connected Stein, Reinhardt domain containing $R$;

if $G / K$ is not of tube type, then $\widehat{D}=K \cdot \widetilde{R}$, where $\widetilde{R}$ is the smallest connected and complete Stein, Reinhardt domain containing $R$.

If $G / K$ is of tube type and $R$ is connected, i.e. $R$ intersects the diagonal line in $\mathbb{C}^{r}$, then by [3], Thm. 2.12, $\widetilde{R}$ coincides with the envelope of holomorphy $\widehat{R}$ of $R$. Similarly, if $G / K$ is not of tube type, then $\widetilde{R}=\widehat{R}$ whenever $R$ is connected and intersects the coordinate hyperplanes.

Our next goal is to extend the characterization of smooth, $K$-invariant, strictly plurisubharmonic functions on $D$ obtained in Theorem 4.5 to the following classes of $K$-invariant functions:

- $P(D)^{K}$ : plurisubharmonic, $K$-invariant functions on $D$,

- $P^{\infty}(D)^{K}$ : smooth, plurisubharmonic, $K$-invariant functions on $D$,

- $P^{+}(D)^{K}$ : functions which, on every relatively compact $K$-invariant domain $C$ in $D$, are the sum $g+h$, of some $g \in P(C)^{K}$ and $h \in P^{\infty,+}(C)^{K}$.

In order to do that we need to define the corresponding appropriate classes of functions on the associated domain $\mathcal{D}_{\mathfrak{a}}$ :

- $\log \operatorname{Conv}\left(\mathcal{D}_{\mathfrak{a}},[-\infty, \infty)\right)^{W}:$ limits of decreasing sequences in $\log \operatorname{Conv} v^{\infty,+}\left(\mathcal{D}_{\mathfrak{a}}\right)^{W}(\mathrm{cf}$. (21)),

$-\log \operatorname{Conv}^{\infty}\left(\mathcal{D}_{\mathfrak{a}}\right)^{W}:$ smooth functions in $\log \operatorname{Conv}\left(\mathcal{D}_{\mathfrak{a}},[-\infty, \infty)\right)^{W}$,

$-\log \operatorname{Conv}^{+}\left(\mathcal{D}_{\mathfrak{a}},[-\infty, \infty)\right)^{W}$ : functions which, on every relatively compact $W$-invariant domain $\mathcal{C}$ of $\mathcal{D}_{\mathfrak{a}}$, are the sum $\tilde{g}+\tilde{h}$ of some $\tilde{g} \in \log \operatorname{Conv}(\mathcal{C},[-\infty, \infty))^{W}$ and $\tilde{h} \in \log \operatorname{Conv}^{\infty,+}(\mathcal{C})^{W}$.

Remark 4.10 (i) The class $\log \operatorname{Conv}^{\infty}\left(\mathcal{D}_{\mathfrak{a}}\right)^{W}$ coincides with the family of smooth $W$ invariant functions on $\mathcal{D}_{\mathfrak{a}}$ for which the form in (ii) of Proposition 3.1 is positive semidefinite. One inclusion is clear. Conversely, if $\tilde{f}$ is smooth and the form in (21) is positive semidefinite, then $\tilde{f}$ is the limit of the sequence $\tilde{f}_{n}\left(a_{1}, \ldots a_{r}\right)=\tilde{f}\left(a_{1}, \ldots a_{r}\right)+$ $\frac{1}{n} \sum a_{j}^{2}$. Hence $\tilde{f}$ belongs to $\log \operatorname{Conv}^{\infty}\left(\mathcal{D}_{\mathfrak{a}}\right)^{W}$. In particular

$$
\log \operatorname{Conv}^{\infty,+}\left(\mathcal{D}_{\mathfrak{a}}\right)^{W} \subset \log \operatorname{Conv}^{\infty}\left(\mathcal{D}_{\mathfrak{a}}\right)^{W} .
$$


(ii) The class $P^{+}(D)^{K}$ coincides with the family of $K$-invariant functions which are locally the sum of some $g$ plurisubharmonic and $h$ smooth strictly plurisubharmonic, i.e. the strictly plurisubharmonic functions according to the definition in $[8$, Def. 1 , Sect. L, p. 118]. Indeed, assume that $f$ is $K$-invariant and strictly plurisubharmonic according to such a definition. Fix a $K$-invariant, smooth strictly plurisubharmonic function $\psi$ on $D$ and let $C$ be a relatively compact $K$-invariant domain of $D$. Then there exists $\varepsilon>0$ such that $g:=f-\varepsilon \psi$ is plurisubharmonic on $C$. That is, $f=g+\varepsilon \psi$, with $g$ psh and $K$-invariant on $C$.

The following lemma shows that all functions in the above classes are continuous.

\section{Lemma 4.11 Let $R$ be a Reinhardt domain.}

(i) Any $T$-invariant plurisubharmonic function $f$ on $R$ is continuous. Its pluripolar set is the union of the intersections of $R$ with some coordinate subspaces.

(ii) The class $\log \operatorname{Conv}\left(\mathcal{D}_{\mathfrak{a}},[-\infty, \infty)\right)^{W}$ is contained in $C^{0}\left(\mathcal{D}_{\mathfrak{a}},[-\infty, \infty)\right)^{W}$.

Proof (i) First consider the case $r=1$. On $R^{*}=R \backslash\{0\}$ one has $f(z)=\hat{f}(\log |z|)$, with $\hat{f}$ convex. Hence the restriction of $f$ to $R^{*}$ is continuos. As $f$ is subharmonic, if $0 \in R$, then one has $f(0)=\limsup _{z \rightarrow 0} f(z)$. Assume by contradiction that

$$
\liminf _{z \rightarrow 0} f(z)<f(0) \text {. }
$$

Then there exists $z_{1} \in R$ close to the origin such that $f\left(z_{1}\right)<f(0)$. By the submean value property and the $S^{1}$-invariance of $f$ one has

$$
f(0) \leq \frac{1}{2 \pi} \int_{0}^{2 \pi} f\left(e^{i \theta} z_{1}\right) d \theta=f\left(z_{1}\right)<f(0),
$$

which is a contradiction.

Let $r=2$. The logarithmic convexity of $f$ on $R^{*}=R \cap\left(\Delta^{*}\right)^{r}$ implies its continuity therein. It remains to prove its continuity on the coordinate lines $\{(z, w) \in R: z w=0\}$ (on each line $f$ can be constant and equal to $-\infty$ ). Assume by contradiction that there exists $\left(z_{0}, 0\right) \in R$ such that

$$
\limsup _{(z, w) \rightarrow\left(z_{0}, 0\right)} f(z, w)-\liminf _{(z, w) \rightarrow\left(z_{0}, 0\right)} f(z, w)>\varepsilon>0 .
$$

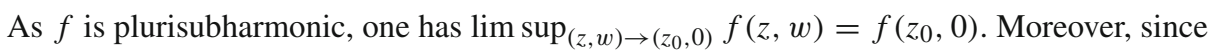
$f$ is continuous on the line $w=0$, there exists a neighborhood $B$ of $z_{0}$ in $\mathbb{C}$ such that

$$
\limsup _{(z, w) \rightarrow\left(z_{0}, 0\right)} f(z, w)-\varepsilon / 2=f\left(z_{0}, 0\right)-\varepsilon / 2<f(\zeta, 0),
$$

for every $\zeta \in B$. By (28), we can choose $\left(\zeta_{1}, w_{1}\right)$ close to $\left(z_{0}, 0\right)$ such that $\zeta_{1} \in B$ and

$$
f\left(\zeta_{1}, w_{1}\right)<\liminf _{(z, w) \rightarrow\left(z_{0}, 0\right)} f(z, w)+\varepsilon / 2<f\left(z_{0}, 0\right)-\varepsilon / 2<f\left(\zeta_{1}, 0\right) .
$$

Then, by the submean value property for subharmonic functions and by the $T$-invariance of $f$ one has

$$
f\left(\zeta_{1}, 0\right) \leq \frac{1}{2 \pi} \int_{0}^{2 \pi} f\left(\zeta_{1}, e^{i \theta} w_{1}\right) d \theta=f\left(\zeta_{1}, w_{1}\right)<f\left(\zeta_{1}, 0\right),
$$

giving a contradiction.

The above argument also shows that the pluripolar set of $f$ consists of either the origin, or of the intersection of $R$ with one or both the coordinate lines. 
By proceeding inductively, one obtains the statement for $r>2$.

(ii) By Theorem 4.5, to a decreasing sequence $\tilde{f}_{n}$ of functions in $\log \operatorname{Conv} v^{\infty,+}\left(\mathcal{D}_{\mathfrak{a}}\right)^{W}$ there corresponds a decreasing sequence $f_{n}$ in $P^{\infty,+}(D)^{K}$, whose limit $f$ necessarily belongs to $P(D)^{K}$. The restriction $\left.f\right|_{R}$ of $f$ to $R$ is a plurisubharmonic $T$-invariant function. By part (i), the function $\left.f\right|_{R}$ is continuous. Consequently so is the corresponding $\tilde{f}$ in $\log \operatorname{Conv}\left(\mathcal{D}_{\mathfrak{a}},[-\infty, \infty)\right)^{W}$, which is the limit of the $\tilde{f}_{n}$.

Summarizing, the following inclusions hold true

$$
\begin{array}{ccccc}
\log _{\operatorname{Conv}}^{+}\left(\mathcal{D}_{\mathfrak{a}},[-\infty, \infty)\right)^{W} & \subset \log \operatorname{Conv}\left(\mathcal{D}_{\mathfrak{a}},[-\infty, \infty)\right)^{W} & \subset C^{0}\left(\mathcal{D}_{\mathfrak{a}},[-\infty, \infty)\right)^{W} \\
\cup & & \cup & \cup & \cup \\
\log \operatorname{Conv}^{\infty,+}\left(\mathcal{D}_{\mathfrak{a}}\right)^{W} & \subset & \log C o n v^{\infty}\left(\mathcal{D}_{\mathfrak{a}}\right)^{W} & \subset & C^{\infty}\left(\mathcal{D}_{\mathfrak{a}}\right)^{W} .
\end{array}
$$

Our complete result is stated in the next theorem.

Theorem 4.12 Let D be a Stein K-invariant domain in an irreducible non-compact Hermitian symmetric space $G / K$. The map $f \rightarrow \tilde{f}$ is a bijection between the following classes of functions

(i) $P^{\infty,+}(D)^{K}$ and $\log \operatorname{Conv}^{\infty,+}\left(\mathcal{D}_{\mathfrak{a}}\right)^{W}$,

(ii) $P(D)^{K}$ and $\log \operatorname{Conv}\left(\mathcal{D}_{\mathfrak{a}},[-\infty, \infty)\right)^{W}$,

(iii) $P^{\infty}(D)^{K}$ and $\log \operatorname{Conv}^{\infty}\left(\mathcal{D}_{\mathfrak{a}}\right)^{W}$,

(iv) $P^{+}(D)^{K}$ and $\log \operatorname{Conv}^{+}\left(\mathcal{D}_{\mathfrak{a}},[-\infty, \infty)\right)^{W}$.

In particular, from the above inclusions, it follows that the $K$-invariant plurisubharmonic functions on D are continuous.

Proof (i) is the content of Theorem 4.5. By averaging over $K$, a $K$-invariant, plurisubharmonic function on $D$ is the decreasing limit of smooth $K$-invariant, strictly plurisubharmonic functions (cf. [8, Sect. K]). Then (ii) follows from (i). As smooth $K$-invariant functions on $D$ correspond to smooth $W$-invariant functions on $\mathcal{D}_{\mathfrak{a}}$, an analogous argument also proves statement (iii). Finally (iv) follows from the definitions of $\operatorname{LogConv} v^{+}(\mathcal{D})^{W}$ and $P^{+}(D)^{K}$, by averaging the summands over $W$ and $K$, respectively.

Let $T \ltimes \mathcal{S}_{r}$ act on $\Delta^{r}$ as in Corollary 4.6. The previous theorem can be reformulated as follows.

Theorem 4.13 Let $D$ be a Stein $K$-invariant domain in an irreducible non-compact Hermitian symmetric space $G / K$ and let $R$ be the associated Reinhardt domain. The map $\left.f \rightarrow f\right|_{R}$ is a bijection between

(i) $P^{\infty,+}(D)^{K}$ and $P^{\infty,+}(R)^{T \ltimes \mathcal{S}_{r}}$,

(ii) $P(D)^{K}$ and $P(R)^{T \ltimes \mathcal{S}_{r}}$,

(iii) $P^{\infty}(D)^{K}$ and $P^{\infty}(R)^{T \ltimes \mathcal{S}_{r}}$,

(iv) $P^{+}(D)^{K}$ and $P^{+}(R)^{T \ltimes \mathcal{S}_{r}}$.

\section{Appendix: A $K$-invariant potential of the Killing metric.}

Let $G / K$ be an irreducible non-compact Hermitian symmetric space. The Killing form $B$ of $\mathfrak{g}$, restricted to $\mathfrak{p}$, induces a $G$-invariant Kähler metric on $G / K$, which we refer to as the Killing metric. In this section we exhibit a $K$-invariant potential $\rho$ of this metric in a Lie 
theoretical fashion. We also show that such a $K$-invariant potential coincides, up to an additive constant, with the logarithm of the Bergman kernel function (Remark 5.2 and Corollary 5.3).

In order to define $\rho$, according to the decomposition $G=K \exp \mathfrak{a} K$, write an element of $G / K$ as $k a K$, where $k \in K$ and $a=\exp H$, with $H=\sum_{j} a_{j} A_{j} \in \mathfrak{a}$.

Proposition 5.1 Let $\widehat{\rho}$ be a real valued function satisfying $\hat{\rho}^{\prime}(t)=\frac{\cosh t-1}{\sinh t}$. Then

(i) the $K$-invariant function $\rho: G / K \rightarrow \mathbb{R}$ defined by

$$
\rho(k a K):=\frac{1}{4} \sum_{j=1}^{r} \widehat{\rho}\left(2 a_{j}\right) B\left(A_{j}, A_{j}\right),
$$

is a potential of the Killing metric;

(ii) the moment map $\mu: G / K \rightarrow \mathfrak{k}^{*}$ associated with $\rho$ is given by

$$
\mu(k a K)(X)=\frac{1}{2} \sum_{j=1}^{r} \sinh \left(2 a_{j}\right) \widehat{\rho}^{\prime}\left(2 a_{j}\right) B\left(\operatorname{Ad}_{k^{-1}} X, K^{j}\right),
$$

where $X \in \mathfrak{k}$.

Proof We first prove (ii). Resume the notation of Section 3. In the proof of Proposition 3.1, it was shown that for $z=a K$ one has

$$
d^{c} \rho\left(\tilde{X}_{z}\right)=0,
$$

for all $X \in \mathfrak{m} \oplus \bigoplus_{\substack{\alpha \in \Sigma^{+} \\ \alpha \neq 2 e_{j}}} \mathfrak{k}[\alpha]$. Moreover, since $\widetilde{\rho}(H)=\frac{1}{4} \sum_{j=1}^{r} \widehat{\rho}\left(2 a_{j}\right) B\left(A_{j}, A_{j}\right)$, from (17) it follows that for $K^{j} \in \mathfrak{k}\left[2 e_{j}\right]$ one has

$$
d^{c} \rho\left(\widetilde{K}^{j}{ }_{z}\right)=-\frac{1}{2} \sinh \left(2 a_{j}\right) \widehat{\rho}^{\prime}\left(2 a_{j}\right) B\left(A_{j}, A_{j}\right) .
$$

As $B\left(A_{j}, A_{j}\right)=-B\left(K^{j}, K^{j}\right)$, then (29), (11) and (16) imply (ii).

(i) We are going to show that on $\mathfrak{p} \times \mathfrak{p}$

$$
h_{\rho}(\cdot, \cdot)=B(\cdot, \cdot) \text {, }
$$

where $h_{\rho}(\cdot, \cdot)=-d d^{c} \rho\left(\cdot, I_{0} \cdot\right)$. By the $K$-invariance of $\rho$ and the orthogonality relations proved in Proposition 3.1, it is sufficient to show that $h_{\rho}\left(a_{*} P, a_{*} Q\right)=B(P, Q)$, for $P, Q$ both in one of the blocks $a_{*} \mathfrak{a}, a_{*} \mathfrak{p}\left[e_{j}+e_{l}\right]$ and $a_{*} \mathfrak{p}\left[e_{j}\right]$.

The form $h_{\rho}$ on $a_{*} \mathfrak{a}$.

Let $A_{j}, A_{l} \in \mathfrak{a}$, be as in (2). Then, by (15) and (10),

$$
\begin{aligned}
h_{\rho}\left(A_{j}, A_{l}\right) & =-d d^{c} \rho\left(a_{*} A_{j}, a_{*} I_{0} A_{l}\right)=-d d^{c} \rho\left(a_{*} P^{l}, a_{*} A_{j}\right) \\
& =\frac{1}{\sinh \left(2 a_{l}\right)} d d^{c} \rho\left(\left(\widetilde{K^{l}}\right)_{z},\left(\widetilde{A_{j}}\right)_{z}\right)=-\left.\frac{1}{\sinh \left(2 a_{l}\right)} \frac{d}{d s}\right|_{s=0} \mu^{K^{l}}\left(\exp s A_{j} \cdot z\right),
\end{aligned}
$$

where $P^{l} \in \mathfrak{p}\left[2 e_{l}\right]$ and $K^{l} \in \mathfrak{k}\left[2 e_{l}\right]$ are as in (3). By (ii), (29) and (11), such quantity vanishes if $l \neq j$. For $j=l$, from the assumption $\widehat{\rho}^{\prime}(t)=\frac{\cosh t-1}{\sinh t}$, we obtain

$$
\begin{aligned}
h_{\rho}\left(A_{l}, A_{l}\right) & =\left.\frac{1}{\sinh \left(2 a_{l}\right)} \frac{1}{2} \frac{d}{d s}\right|_{s=0} \sinh \left(2 a_{l}+2 s\right) \widehat{\rho}^{\prime}\left(2 a_{l}+2 s\right) B\left(A_{l}, A_{l}\right) \\
& =\frac{1}{\sinh \left(2 a_{l}\right)}\left(\cosh \left(2 a_{l}\right) \widehat{\rho}^{\prime}\left(2 a_{l}\right)+\sinh \left(2 a_{l}\right) \widehat{\rho}^{\prime \prime}\left(2 a_{l}\right)\right) B\left(A_{l}, A_{l}\right)=B\left(A_{l}, A_{l}\right),
\end{aligned}
$$

as desired. 
Let $\alpha$ be a root in $\Sigma^{+} \backslash\left\{2 e_{j}\right\}$ and let $P=X-\theta X \in \mathfrak{p}[\alpha]$, for some $X \in \mathfrak{g}^{\alpha}$. Then $Q:=I_{0} P=Y-\theta Y \in \mathfrak{p}[\beta]$, for some $Y \in \mathfrak{g}^{\beta}$ with $\beta \in \Sigma^{+}$. Define $K:=X+\theta X \in \mathfrak{k}[\alpha]$ and $C:=Y+\theta Y \in \mathfrak{k}[\beta]$. As $K^{j}=\left[I_{0} A_{j}, A_{j}\right]$, by (18) and (ii),

$$
\begin{aligned}
h_{\rho}\left(a_{*} P, a_{*} P\right) & =-\frac{1}{\sinh \alpha(H) \sinh \beta(H)} \frac{1}{2} \sum_{k} \sinh \left(2 a_{k}\right) \hat{\rho}^{\prime}\left(2 a_{k}\right) B\left([C, K],\left[I_{0} A_{k}, A_{k}\right]\right) \\
& =-\frac{1}{\sinh \alpha(H) \sinh \beta(H)} \frac{1}{2} \sum_{k} \sinh \left(2 a_{k}\right) \hat{\rho}^{\prime}\left(2 a_{k}\right) B\left(K,\left[\left[I_{0} A_{k}, A_{k}\right], C\right]\right) .
\end{aligned}
$$

From the Jacobi identity, one has

$$
\begin{aligned}
B\left(K,\left[\left[I_{0} A_{k}, A_{k}\right], C\right]\right) & =-B\left(K,\left[\left[C, I_{0} A_{k}\right], A_{k}\right]+\left[\left[A_{k}, C\right], I_{0} A_{k}\right]\right) \\
& =B\left(\left[A_{k}, K\right],\left[I_{0} A_{k}, C\right]\right)-B\left(\left[I_{0} A_{k}, K\right],\left[A_{k}, C\right]\right) \\
& =\alpha\left(A_{k}\right) B\left(P, I_{0}\left[A_{k}, C\right]\right)-\beta\left(A_{k}\right) B\left(I_{0}\left[A_{k}, K\right], Q\right) \\
& =\left(\alpha\left(A_{k}\right) \beta\left(A_{k}\right)+\beta\left(A_{k}\right) \alpha\left(A_{k}\right)\right) B\left(P, I_{0} Q\right) .
\end{aligned}
$$

As $I_{0} Q=-P$, one obtains

$$
\begin{aligned}
h_{\rho}\left(a_{*} P, a_{*} P\right)= & \frac{1}{2 \sinh \alpha(H) \sinh \beta(H)} \sum_{k} \sinh \left(2 a_{k}\right) \widehat{\rho}^{\prime}\left(2 a_{k}\right)\left(\alpha\left(A_{k}\right) \beta\left(A_{k}\right)\right. \\
& \left.+\beta\left(A_{k}\right) \alpha\left(A_{k}\right)\right) B(P, P) .
\end{aligned}
$$

We are left to specialize the above formula in the cases $\alpha=e_{j}+e_{l}$ and $\alpha=e_{j}$, for $j, l=1, \ldots, r$.

The form $h_{\rho}$ on $a_{*} \mathfrak{p}\left[e_{j}+e_{l}\right]$.

Here $\alpha=e_{j}+e_{l}$ and $\beta=e_{j}-e_{l}$. Then for $P \in \mathfrak{p}\left[e_{j}+e_{l}\right]$, one has

$$
\begin{aligned}
h_{\rho}\left(a_{*} P, a_{*} P\right) & =\frac{1}{2 \sinh \left(a_{j}+a_{l}\right) \sinh \left(a_{j}-a_{l}\right)}\left(\sinh \left(2 a_{j}\right) \hat{\rho}^{\prime}\left(2 a_{j}\right)-\sinh \left(2 a_{l}\right) \hat{\rho}^{\prime}\left(2 a_{l}\right)\right) B(P, P) \\
& =\frac{\cosh \left(2 a_{j}\right)-\cosh \left(2 a_{l}\right)}{2 \sin \left(a_{j}+a_{l}\right) \sin \left(a_{j}-a_{l}\right)} B(P, P)=B(P, P),
\end{aligned}
$$

due to the identity $\cosh \left(2 a_{j}\right)-\cosh \left(2 a_{l}\right)=2 \sinh \left(a_{j}+a_{l}\right) \sinh \left(a_{j}-a_{l}\right)$.

The form $h_{\rho}$ on $a_{*} \mathfrak{p}\left[e_{j}\right]$.

Here $\alpha=\beta=e_{j}$. Then for $P \in \mathfrak{p}\left[e_{j}\right]$, one has

$$
\begin{aligned}
h_{\rho}\left(a_{*} P, a_{*} P\right) & =\frac{1}{2 \sinh ^{2}\left(a_{j}\right)} \sinh \left(2 a_{j}\right) \hat{\rho}^{\prime}\left(2 a_{j}\right) B(P, P) \\
& =\frac{1}{2 \sinh ^{2}\left(a_{j}\right)}\left(\cosh \left(2 a_{j}\right)-1\right) B(P, P)=B(P, P) .
\end{aligned}
$$

This concludes the proof of (i) and of the proposition.

The following remark shows that a $K$-invariant potential of the Killing metric is unique, up to an additive constant.

Remark 5.2 Let $\rho_{1}$ and $\rho_{2}$ be smooth $K$-invariant functions on $G / K$ such that $d d^{c} \rho_{1}=$ $d d^{c} \rho_{2}$. Then $\rho_{1}-\rho_{2}$ is constant.

Proof As $\rho_{1}-\rho_{2}$ is pluriharmonic and $G / K$ is contractible, there exists a holomorphic function $f: G / K \rightarrow \mathbb{C}$, such that $\operatorname{Re} f=\rho_{1}-\rho_{2}$, which is unique up to an imaginary constant (cf. [8, Sect. K]). By averaging $f$ over $K$, its real part $\rho_{1}-\rho_{2}$ does not change. Hence $f$ itself is $K$-invariant. Moreover, being holomorphic, $f$ is also invariant with respect to the induced local $K^{\mathbb{C}}$-action on $G / K$. Since $K^{\mathbb{C}}$ acts locally transitively on an open subset of $G / K$ (cf. [13]), the function $f$ is constant and so is its real part $\rho_{1}-\rho_{2}$. 
Since the logarithm of the Bergman kernel function is a $K$-invariant potential of the Killing metric (see [10], Vol.2, Exa.6.6, p. 162 and Thm.9.6, p. 262), one can draw the following conclusion.

Corollary 5.3 Up to an addictive constant, the smooth $K$-invariant exhaustion function $\rho$ coincides with the logarithm of the Bergman kernel function.

Example 5.4 As an example, consider the unit disc $\Delta=G / K$, where $G=S U(1,1)$ acts on $\Delta$ by linear fractional transformations. Fix the basis of $\mathfrak{g}$, normalized as in (3):

$$
K^{1}=\left(\begin{array}{cc}
i & 0 \\
0 & -i
\end{array}\right), \quad A_{1}=\left(\begin{array}{ll}
0 & 1 \\
1 & 0
\end{array}\right), \quad P^{1}=\left(\begin{array}{cc}
0 & -i \\
i & 0
\end{array}\right) .
$$

Then $\exp a_{1} A_{1} K=\tanh a_{1}=|z|$. Take $\widehat{\rho}(t)=-\ln \frac{1}{\cosh t+1}$, which satisfies the differential equation $\hat{\rho}^{\prime}(t)=\frac{\cosh t-1}{\sinh t}$. Since $B\left(A_{1}, A_{1}\right)=8$, then up to an addictive constant, the logarithm of the Bergman kernel function is given by

$$
\begin{aligned}
\rho\left(\exp a_{1} A_{1} K\right) & =\quad-\frac{1}{4} \ln \frac{1}{\cosh 2 a_{1}+1} B\left(A_{1}, A_{1}\right) \\
& =-2 \ln \frac{\cosh ^{2} a_{1}-\sinh ^{2} a_{1}}{2 \cosh ^{2} a_{1}}=-2 \ln \left(1-|z|^{2}\right)+\text { const } .
\end{aligned}
$$

Funding Open access funding provided by Università degli Studi di Roma Tor Vergata within the CRUI-CARE Agreement.

Open Access This article is licensed under a Creative Commons Attribution 4.0 International License, which permits use, sharing, adaptation, distribution and reproduction in any medium or format, as long as you give appropriate credit to the original author(s) and the source, provide a link to the Creative Commons licence, and indicate if changes were made. The images or other third party material in this article are included in the article's Creative Commons licence, unless indicated otherwise in a credit line to the material. If material is not included in the article's Creative Commons licence and your intended use is not permitted by statutory regulation or exceeds the permitted use, you will need to obtain permission directly from the copyright holder. To view a copy of this licence, visit http://creativecommons.org/licenses/by/4.0/.

\section{References}

1. Azad, H., Loeb, J.J.: Plurisubharmonic functions and the Kempf-Ness theorem. Bull. Lond. Math. Soc. 25, 162-168 (1993)

2. Bedford, E., Dadok, J.: Generalized Reinhardt domains. J. Geom. Anal. 1, 1-17 (1991)

3. Carmignani, R.: Envelopes of holomorphy and holomorphic convexity. Trans. AMS 179, 415-431 (1973)

4. Dadok, J.: On the $C^{\infty}$ Chevalley theorem. Adv. Math. 44, 121-131 (1982)

5. Fels, G., Huckleberry, A.T.: A characterization of K-invariant Stein Domains in Symmetric Embeddings. In: Complex Analysis and Geometry, pp. 223-234. Univ. Ser. Math., Plenum, New York (1993)

6. Flensted-Jensen, M.: Spherical functions of real semisimple Lie groups. A method of reduction to the complex case. J. Funct. Anal. 30, 106-146 (1978)

7. Geatti, L., Iannuzzi, A.: Orbit structure of a distinguished invariant domain in the complexification of a Hermitian symmetric space. Math. Zeit. 278(3-4), 769-793 (2014)

8. Gunning, R.C.: Introduction to Holomorphic Functions of Several Variables, vol. I, Function Theory. Wadsworth \& Brooks/Cole (1990)

9. Heinzner, P., Schwarz, G.W.: Cartan decomposition of the moment map. Math. Ann. 337, 197-232 (2007)

10. Kobayashi, S., Nomizu, K.: Foundations of Differential Geometry, vol. 2. Interscience Tracts in Pure and Applied Mathematics, No. 15. Wiley, New York, London, Sydney (1969)

11. Rossi, H., Vergne, M.: Representations of certain solvable Lie groups on Hilbert spaces of holomorphic functions and the application to the holomorphic discrete series of a semisimple Lie group. J. Funct. Anal. 13, 324-389 (1973)

12. Satake, I.: Algebraic Structures of Symmetric Domains. Princeton University Press, Princeton (1980) 
13. Wolf, J.A.: Fine structure of Hermitian symmetric spaces. In: Boothby, W., Weiss, G. (eds.) Symmetric spaces (Short Courses, Washington University), Pure and Applied Mathematics, vol. 8, pp. 271-357. Dekker, New York (1972)

Publisher's Note Springer Nature remains neutral with regard to jurisdictional claims in published maps and institutional affiliations. 